Alma Mater Studiorum - Università di Bologna DEPARTMENT OF ECONOMICS

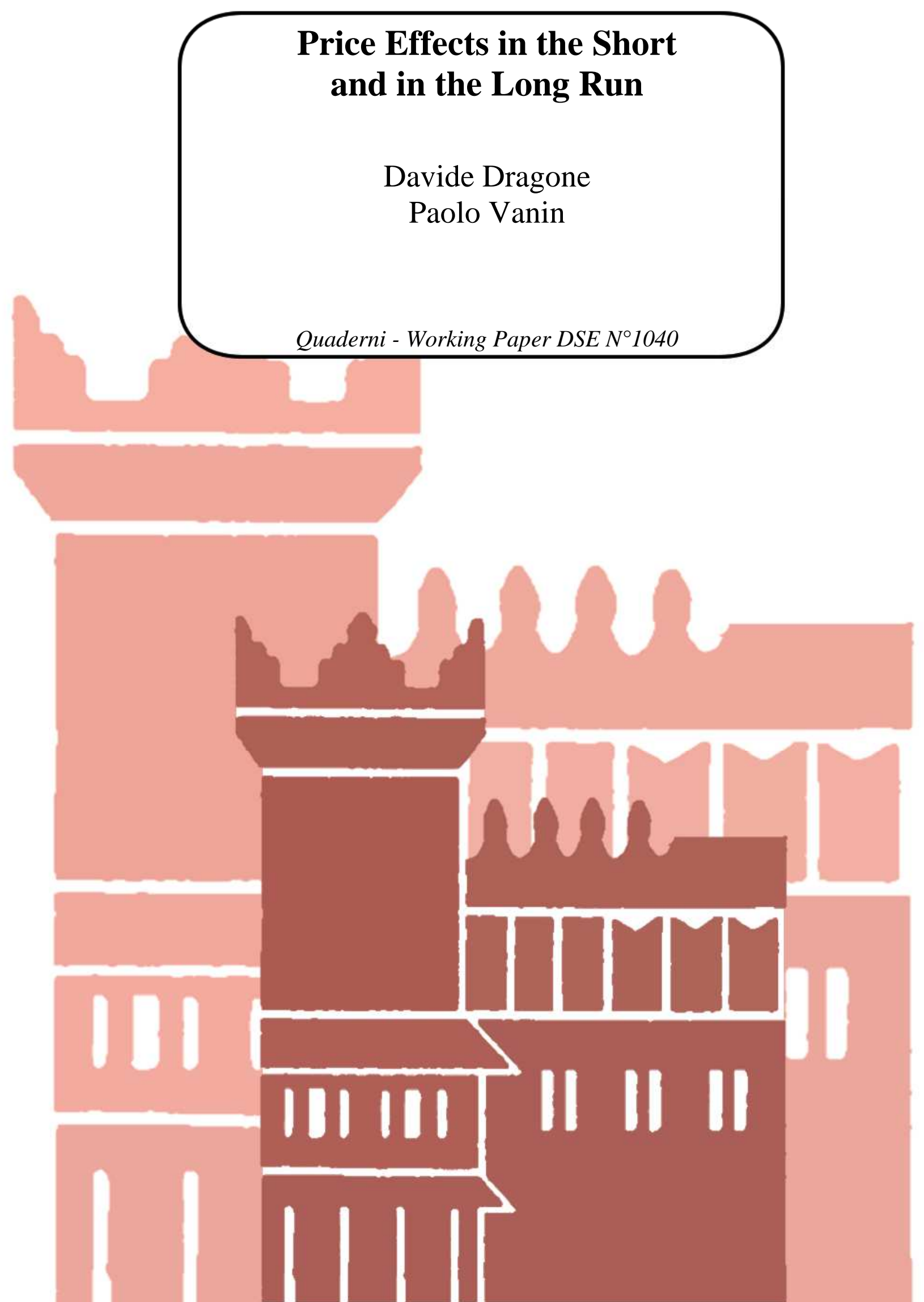




\title{
Price Effects in the Short and in the Long Run*
}

\author{
Davide Dragone \\ Paolo Vanin \\ University of Bologna ${ }^{\dagger}$ \\ University of Bologna
}

November 27, 2015

\begin{abstract}
We study a broad class of dynamic consumer problems and characterize the short and long-run response of the demand for a good to a permanent increase in its market price. Such response can be non-monotonic over time, and the short and long-run price-elasticity of demand may have opposite sign. This is a testable prediction and can arise even in the absence of income effects. Our results are robust to a variety of settings that are commonly used in the economic literature, and have relevant policy implications. We provide illustrative applications to models of human capital and labor supply, addiction, habit and taste formation, health capital, and renewable resources.
\end{abstract}

JEL-Classification: D11, D91, I00

Keywords: Price elasticity, Non-monotonicity, Price-based policies

*We thank for useful comments and suggestions Antonio Cabrales, Vincenzo Denicolò, Antonio Minniti, Tito Pietra and the participants to the 2015 ASSET Conference in Granada, the 2015 EEA Congress in Mannheim, the 2015 UPF-GPEFM Alumni Meeting in Barcelona, and the Economics Department Seminar in Bologna. The usual disclaimer applies.

${ }^{\dagger}$ University of Bologna, Department of Economics, Piazza Scaravilli 2, 40126, Bologna, Italy; phone: +39-0512098880; e-mail: davide.dragone@unibo.it

$\ddagger$ University of Bologna, Department of Economics, Piazza Scaravilli 2, 40126, Bologna, Italy; phone: +39-0512098120; e-mail: paolo.vanin@unibo.it 


\section{Introduction}

Consider a subsidy to schooling financed through labor income taxes. Will households respond to its introduction by investing more in human capital accumulation due to the current lower opportunity cost of schooling, or will they invest less because they anticipate the lower future returns to human capital? Is it possible that schooling increases in the short run but decreases in the long run, or the other way around? Under which conditions does this happen? Would such policy eventually result in higher or lower human capital?

Answering this kind of questions is not only relevant for explaining dynamic consumer behavior in situations that involve a trade-off between short and long run, as it is commonly the case in areas ranging from macro to public economics, from labor to health economics, and from cultural to environmental economics, but it is also relevant for an appropriate design of price-based policies, which may in principle produce opposite consumer behavior in the short and in the long run, and may generate non-obvious dynamic effects on stock variables such as human capital, health or natural resources, whose long-run externalities are often the motivation for policy intervention.

More generally, while price effects in static consumer theory are well established, less is known about short and long-run price effects in dynamic consumer theory. We provide a theoretical answer to the following simple but fundamental questions. Do standard static results, such as the validity of the law of demand for non-Giffen goods, extend to a dynamic framework? Do they hold over different time horizons? Has the elasticity of demand the same sign in the short as in the long run? Is demand more rigid in the short than in the long run?

To address these questions, we study a broad and abstract class of dynamic consumer problems, and we characterize the short and long-run response of the demand for a good to a permanent increase in its market price. The class of problems we consider allows for the effect of past actions on current choices, for either static or dynamic budget constraints, and for resource allocation to consumption goods as well as for time allocation to labor and leisure. Our results do not rely on specific functional forms but rather depend on the general dynamic structure of intertemporal consumer problems.

We show that price effects have two components, which are the dynamic counterparts of income and substitution effects in static problems. As it is true in a static framework, income effects can lead to violations of the law of demand. Differently from a static frame-

work, however, the dynamic counterpart of the substitution effect can lead by itself to violations of the law of demand, which can arise even in the absence of income effects.

As a permanent price increase modifies the optimal path of consumption, we say that the law of demand holds at a given time if the quantity demanded at that time is lower 
along the new than along the old optimal path.

We establish conditions under which consumer response to price change is non-monotonic over time, and the law of demand holds in the short run, but is violated in the long run, or the other way around. We also show conditions under which the law of demand holds both in the short and in the long run. Even when this is the case, we find that demand can respond non monotonically over time and can be more elastic in the short than in the long run, contrary to common intuition.

Despite the complexity of dynamic models, the above conditions can be surprisingly simple. Under a set of assumptions that are common in the economic literature, we show that the sign of price effects only depends on the relation between impatience and state dynamics: the dynamic technology of the problem determines how current choices generate future consequences, and time preferences determine the subjective balance between present and future. If the future consequences of current behavior are amplified over time, as it easily happens in models of human capital and renewable resources, we have the nonmonotonicity result whereby the short and long-run elasticity of demand have opposite sign, which means that the law of demand is violated at some point in time. In particular, for patient consumers the law of demand is violated in the short run but holds in the long run, whereas for impatient consumers the reverse is true. If instead the future consequences of current behavior are dampened, as it easily happens in models with capital depreciation, a price increase reduces demand at every point in time. Still, in this case there can be overshooting in the short-run relative to the long run, and thus non-monotonicity over time. $^{1}$

The prediction of a non-monotonic response over time is empirically testable with individual level panel data, for instance by looking at exogenous sources of price variation such as taxes and subsidies. If at a given point in time patient and impatient consumers respond in opposite ways to a price change, unobserved heterogeneity in time preferences produces an attenuation bias in estimated price responses, due to a composition effect.

The possibility that the short and long-run elasticity of demand have opposite sign is also relevant for policy and for the interpretation of the empirical evidence on violations of the law of demand. Whether a price-based policy that affects behavior in opposite ways in the short and in the long run is qualitatively 'right' or 'wrong' depends on the time

\footnotetext{
${ }^{1}$ Using the formalization introduced below and denoting by $\rho$ the discount rate and by $f_{S}$ the marginal effect of the state variable $S$ on its own speed of change, an amplification effect obtains if $f_{S}>0$, because an initial behavior that raises $S$ leads to a subsequent increase in the speed of growth of $S$; a stabilization effect instead obtains if $f_{S}<0$. Under a dynamic amplifier, consumers respond to a price increase in opposite ways over time depending on whether they are patient $\left(\rho<f_{S}\right)$ or impatient $\left(\rho>f_{S}\right)$; and if consumption reduces $S$, a price increase triggers a reduction of $S$ over time for patient consumers and an increase of $S$ for impatient ones. Under a dynamic stabilizer, short-run overshooting occurs if $f_{S}$ is not too negative; and if consumption reduces $S$, a price increase triggers an increse of $S$ over time.
} 
horizon over which it is evaluated. Shortsighted policymakers may then adopt policies that seem 'right' in the short run, but prove 'wrong' in the long run. Further, since patient and impatient consumers may react to price changes in opposite ways over time, policies designed for either type of consumer may have undesired consequences for the other type. Finally, since we show that the law of demand can be violated at some point in time even by non-Giffen goods, our results provide a new possible dynamic interpretation for the evidence of violations of the law of demand, which has been so far interpreted as Giffen behavior, among other things, for the lack of a clear alternative theoretical framework. ${ }^{2}$

To present the consumer dynamic behavior in the most transparent way, Section 2 develops an illustrative model that is simple, rules out income effects by construction, and allows for a closed form solution. It generates non-monotonic dynamic responses to a price increase, with short and long run price elasticities of opposite sign. For patient consumers demand initially rises and then falls; eventually, consumption and the state variable reach a level that is lower in the new steady state than in the old one. For impatient consumers the reverse is true. ${ }^{3}$

Sections 3 to 5 generalize the analysis and consider an abstract class of intertemporal consumer problems that encompass the workhorse models used in a variety of fields in economic research, including static and dynamic budget constraint, as well as labor supply. ${ }^{4}$ We characterize price effects on consumption and on the state variable of interest, and we highlight how violations of the law of demand can emerge at different points in time, even in the absence of income effects.

In Section 6 we illustrate the applicability of our results by considering classic intertemporal models of human capital accumulation, addiction, habit and taste formation, health, and exploitation of natural resources (Ben-Porath, 1967; Heckman, 1976; Blinder and Weiss, 1976; Arrow, 1962; Weiss, 1972; Becker and Murphy, 1988; Stigler and Becker, 1977; Abel, 1990; Carroll et al., 2000; Grossman, 1972; Clark et al., 1979). This selection of applications is clearly not exhaustive: it just shows how our results can be applied to a broad range of economic research in different fields, for which intertemporal consumer problems are a building block. Section 7 concludes.

\footnotetext{
${ }^{2}$ For instance, Jensen and Miller (2008) report evidence of a decrease in rice demand in China after a temporary reduction in its price and interpret it as Giffen behavior.

${ }^{3}$ The illustrative model assumes a dynamic amplifier, with $f_{S}=1$, so that patient and impatient consumers are identified by $\rho<1$ and $\rho>1$, respectively.

${ }^{4}$ The comparative statics and comparative dynamics properties of dynamic optimization models have been studied, among others, by Oniki (1973), Epstein (1978), Otani (1982), and Caputo (1990, 1997). Our work is related to this literature, but our focus on consumer problems allows for an explicit analysis of price effects, and our investigation covers classes of models that are not considered in this literature, but are relevant in economic research.
} 


\section{An illustrative example}

In this Section we consider an illustrative dynamic consumer problem that rules out income effects and is simple enough to be solved in close form. This example illustrates three notable features concerning short and long-run price effects in a dynamic consumer problem: (i) price effects can be non-monotonic over time, (ii) price effects can have different sign over different time horizons, and (iii) price increases can lead to an increase in demand at some point in time, thereby violating the law of demand, even in absence of income effects.

Let the instantaneous utility function be defined over two goods $x$ and $y$ and a state variable $S$ :

$$
U(x, y, S)=x-\frac{x^{2}}{2}+y+S-\frac{S^{2}}{2} .
$$

Given a market price $p$, income $M$, an initial state $S_{0}$, and a discount rate $\rho$, the consumer chooses the time path of $x$ and $y$ that solves the following problem:

$$
\begin{aligned}
\max _{\{x, y\}} & \int_{0}^{\infty} e^{-\rho t}\left(x-\frac{x^{2}}{2}+y+S-\frac{S^{2}}{2}\right) \mathrm{d} t \\
\text { s.t. } & M=p x+y \\
& \dot{S}=S-x .
\end{aligned}
$$

We present here the essential elements of the solution, and display full details in Appendix A.1. The corresponding static problem, in which $S$ is taken as given, is analyzed for comparability in Appendix A.2. Replacing the budget constraint, the associated currentvalue Hamiltonian function is

$$
\mathcal{H}(x, S, \mu ; p, M)=\left(x-\frac{x^{2}}{2}+S-\frac{S^{2}}{2}+M-p x\right)+\mu(S-x)
$$

where $\mu$ represents the shadow price of the state variable. Let us focus on the case in which $p, M$ and $S_{0}$ are such that $x$ and $y$ are positive along the optimal path. The necessary and sufficient conditions for an internal solution are:

$$
\begin{aligned}
\mathcal{H}_{x} & =1-x-p-\mu=0 \\
\dot{\mu} & =\mu(\rho-1)+S-1 \\
\dot{S} & =S-x
\end{aligned}
$$

together with the transversality condition $\lim _{t \rightarrow \infty} e^{-\rho t} \mu S=0 .^{5}$ Note that the first order condition (FOC) $\mathcal{H}_{x}=0$ equates the marginal utility of consumption to its full price, which

\footnotetext{
${ }^{5}$ Throughout the paper subscripts denote partial derivatives.
} 
is given by the sum of the market price of $x$ and the shadow value of the state variable, so that $U_{x}=p+\mu$. In the corresponding static problem the relevant FOC simply equates the marginal utility of $x$ to its market price $\left(U_{x}=p\right)$.

Differentiating $\mathcal{H}_{x}=0$ with respect to time and replacing $\mu=1-x-p$ from (5) one can express the necessary and sufficient conditions in terms of the dynamics of the (optimal) control variable $x$ and the state variable $S$ :

$$
\begin{aligned}
& \dot{x}=1-S+(x+p-1)(\rho-1) \\
& \dot{S}=S-x .
\end{aligned}
$$

Setting $\dot{x}=\dot{S}=0$ yields the steady state values of the optimal solution

$$
x^{s s}=S^{s s}=1+\frac{1-\rho}{|J|} p
$$

where $|J|=\rho-2$ is the determinant of the Jacobian matrix $J$ associated to (8) and (9). Saddle point stability requires $|J|<0$, which holds if $\rho \in(0,2)$. The policy function leading to the steady state is

$$
x(S)=x^{s s}+(1-\varepsilon)\left(S-S^{s s}\right)
$$

where $\varepsilon$, which only depends on $\rho$, is the negative eigenvalue of $J$.

Since both the policy function and the related steady state are independent of income $\left(\partial x(S) / \partial M=\partial x^{s s} / \partial M=0\right)$, this simple dynamic consumer problem rules out income effects. ${ }^{6}$ Yet, differently from the static case, the optimal solution displays a non-monotonic dynamic response to price changes, with short and long run price elasticities of opposite sign and the consequent violation of the law of demand at some point in time.

Remark 1 (Dynamic price effect: illustrative example) In a steady state with saddle point stability of problem (2)-(4), a permanent increase in p affects the consumption of $x$ in opposite directions in the short and in the long run:

- when the consumer is sufficiently impatient $(\rho>1)$, consumption of $x$ decreases on impact and increases in the long run;

- when the consumer is sufficiently patient $(\rho<1)$, consumption of $x$ increases on impact and decreases in the long run.

\footnotetext{
${ }^{6}$ In a static problem a quasi-linear utility function is sufficient to rule out income effects. In the corresponding dynamic problem the absence of income effects on $x$ is not only due to the quasi-linear utility function, but also to the dynamics of $S$ being independent of $y$, as shown in the next Section.
} 

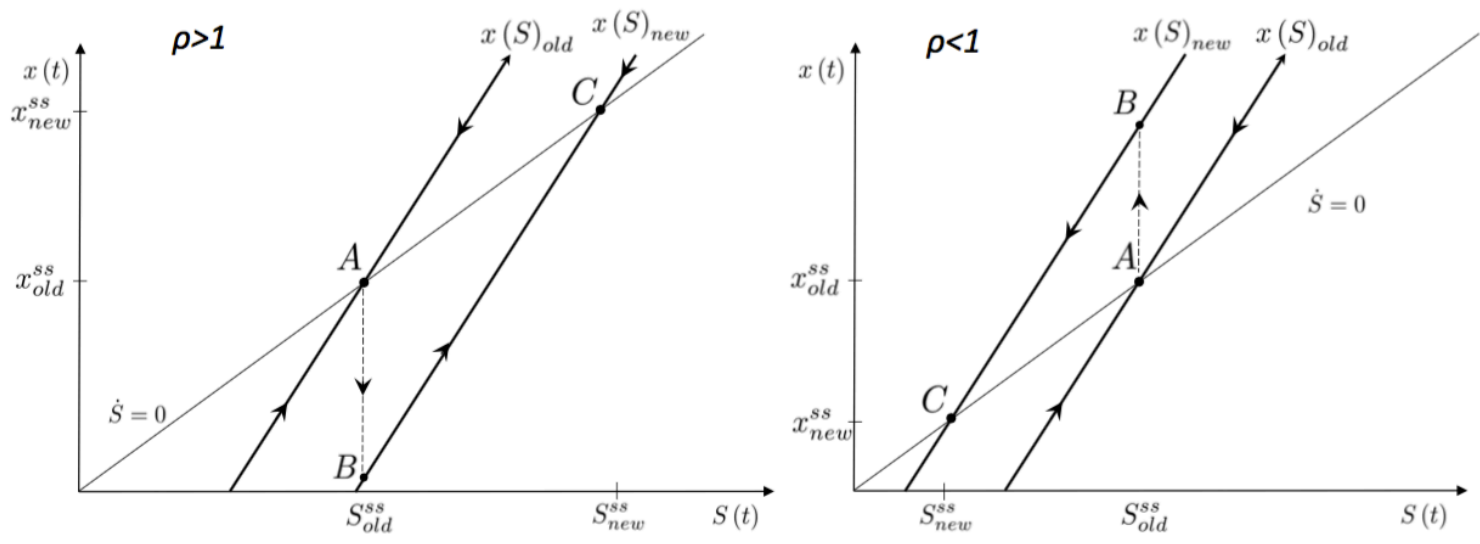

Figure 1: Short and long-run price effects. A permanent price increase makes the policy function $x(S)$ shift downward for an impatient consumer $(\rho>1$, left panel), and upward for a patient consumer $(\rho<1$, right panel). Consumption of $x$ on impact jumps from $A$ to $B$, then it follows the new policy function $x(S)_{\text {new }}$ until the new steady state $C$ is reached.

Figure 1 provides a graphical illustration of Remark 1. It displays the response of $x$ to a permanent increase in its price $p$ occurring at a stable steady state for an impatient consumer (left panel, $\rho>1$ ) and for a more patient consumer (right panel, $\rho<1$ ). In the former case, the policy function shifts downward and the demand for $x$ initially drops, subsequently increases, and eventually reaches a higher level in the new steady state than the initial one. In the latter case the time pattern is reversed.

The economic intuition is the following. In a dynamic environment, a forward looking consumer must balance short and long-run considerations when choosing the optimal consumption path. The balance depends on the intertemporal discount rate $\rho$, among other factors. If the agent is impatient $(\rho>1)$, short run considerations are relatively more important than long run ones. On impact, she behaves as in a static environment where, absent income effects, the substitution effect induces a reduction in $x$ after a rise in $p$. A lower $x$ determines an increase in the state variable $S$, which progressively lowers the full price of $x$ and thus raises its consumption, eventually resulting in a higher steady state level of both $x$ and $S$. An impatient consumer heavily discounts these long run consequences and reacts on impact as she would in a static model.

If instead the agent is patient $(\rho<1)$, long run considerations are relatively more important than short run ones. In steady state, as the full price of $x$ increases when its market price increases, a patient consumer aims at reducing $x$. To achieve this goal, she reacts on impact in a way that is opposite to what she would do in a static environment, by initially consuming more $x$ in order to reduce $S$ over time and thus allow herself a lower consumption of $x$ in the future. 
After showing that impatience, and the related trade-off between short and long run considerations can produce interesting dynamic behavior even in a simple problem, in the following Sections we consider a general set-up where we characterize short and long run price effects without relying on specific functional forms.

\section{A dynamic consumer problem without saving}

Let us now consider a general dynamic consumer maximization problem. In this Section we consider the case with a static budget constraint, which is the simplest dynamic extension of static consumer theory. ${ }^{7}$ In the next Section we relax such constraint and include the possibility of saving, borrowing and lending.

Consider an instantaneous utility function $U(x(t), y(t), S(t))$ and a budget constraint $M+w(S(t)) \geq p x(t)+y(t)$, where $x(t) \geq 0$ and $y(t) \geq 0$ are consumption goods and $S(t)$ is a state variable that may affect utility and income. Good $y$ is the numeraire, $p$ is the market price of good $x, M$ is the exogenous component of income and $w(S)$ is its endogenous (state-dependent) component, assumed to be continuously differentiable and concave. The state variable evolves according to $\dot{S}=f(x(t), y(t), S(t))$. Omitting the time arguments for brevity, we assume that $U(x, y, S)$ and $f(x, y, S)$ are continuously differentiable and concave functions in $(x, y, S)$, with $U_{x x}<0$. We will focus on the case in which the budget constraint is binding and the non-negativity constraints are not.

Given an intertemporal discount rate $\rho>0$ and initial state $S(0)=S_{0}$, the consumer's dynamic problem consists in determining the time path of $x$ and $y$ that solves:

$$
\begin{aligned}
\max _{\{x, y\}} & \int_{0}^{\infty} e^{-\rho t} U(x, y, S) \mathrm{d} t \\
\text { s.t. } & M+w(S)=p x+y \\
& \dot{S}=f(x, y, S) .
\end{aligned}
$$

The corresponding current-value Hamiltonian function is

$$
\mathcal{H}(x, y, \lambda, S, \mu ; p, M)=U(x, y, S)+\lambda[M+w(S)-p x-y]+\mu f(x, y, S)
$$

where $\lambda$ is the Lagrange multiplier of the budget constraint and $\mu$ is the costate variable associated to state $S$. If the Hamiltonian function is concave in state and control variables, the following conditions are necessary and sufficient for an internal solution (Mangasarian,

\footnotetext{
${ }^{7}$ For comparability, the static counterpart of the current model is provided in Appendix A.3.
} 
1966; Seierstad and Sydsaeter, 1977):

$$
\begin{aligned}
\mathcal{H}_{x} & =U_{x}(x, y, S)-\lambda p+\mu f_{x}(x, y, S)=0 \\
\mathcal{H}_{y} & =U_{y}(x, y, S)-\lambda+\mu f_{y}(x, y, S)=0 \\
\mathcal{H}_{\lambda} & =M+w(S)-p x-y=0 \\
\dot{\mu} & =\rho \mu-\mathcal{H}_{S}(x, y, S) \\
\dot{S} & =f(x, y, S)
\end{aligned}
$$

with the transversality condition $\lim _{t \rightarrow \infty} e^{-\rho t} \mu(t) S(t)=0 .^{8}$

Assuming strict concavity of the Hamiltonian function with respect to $x$, the FOCs (16)-(18) determine the optimal value of $x, y$ and $\lambda$ as functions of the state and costate variable, of the market price and the exogenous component of income:

$$
\begin{aligned}
x^{*} & =x^{*}(S, \mu ; p, M) \\
y^{*} & =y^{*}(S, \mu ; p, M) \\
\lambda^{*} & =\lambda^{*}(S, \mu ; p, M) .
\end{aligned}
$$

Replacing $\left(x^{*}, y^{*}, \lambda^{*}\right)$ in (19)-(20) yields the optimal state and costate dynamics:

$$
\begin{aligned}
\dot{\mu} & =\rho \mu-\mathcal{H}_{S}\left(x^{*}, y^{*}, \lambda^{*}, S, \mu ; p, M\right) \\
\dot{S} & =f\left(x^{*}, y^{*}, S\right) .
\end{aligned}
$$

Given the initial state and the transversality condition, the solution $\{S(t), \mu(t)\}$ of the above system of differential equations represents the optimal trajectories of the state and costate variables, which depend on $p$ and $M$ and determines the time-path of $x, y$ and $\lambda$.

We now focus on trajectories leading to a steady state $\left(S^{s s}, \mu^{s s}\right)$, and on the consumption of $x$. For later use, and with a slight abuse of notation, let us write steady state variables as functions of $p$ and $M: S^{s s}=S^{s s}(p, M), \mu^{s s}=\mu^{s s}(p, M)$, and $x^{s s}=x^{s s}(p, M)=$ $x^{*}\left(S^{s s}, \mu^{s s} ; p, M\right)$. Consumer behavior along the optimal trajectories leading to a steady state is captured by a function $\mu(S)$, which, plugged into (21), yields the policy function

$$
x(S)=x^{*}(S, \mu(S) ; p, M)
$$

\footnotetext{
${ }^{8}$ If $f(x, y, S)$ is non linear, the above conditions are necessary and sufficient for a maximum provided along the optimal solution $\mu(t) \geq 0$ for all $t$. The budget constraint is binding if, along the optimal path, $\lambda$ is positive, which occurs when $U_{x}(x, y, S)+\mu f_{x}(x, y, S)>0$ and $U_{y}(x, y, S)+\mu f_{y}(x, y, S)>0$ at all $t$. We focus on this case. An alternative way of solving the problem would be to substitute away the budget constraint and obtain a Hamiltonian function with one control and one state variable, as in the illustrative example and, for instance, in Caputo (1997).
} 
Consider a permanent change in $p$, assuming that the consumer is already in steady state. On impact, its effect on the consumption of $x$ is captured by the change in the policy function (26), given $S=S^{s s}$ :

$$
\frac{\mathrm{d} x\left(S^{s s}\right)}{\mathrm{d} p}=\frac{\partial x^{*}}{\partial p}+\frac{\partial x^{*}}{\partial \mu} \frac{\mathrm{d} \mu\left(S^{s s}\right)}{\mathrm{d} p} .
$$

The change in steady state consumption captures the long-run effect:

$$
\frac{\partial x^{s s}}{\partial p}=\frac{\partial x^{*}}{\partial p}+\frac{\partial x^{*}}{\partial S} \frac{\partial S^{s s}}{\partial p}+\frac{\partial x^{*}}{\partial \mu} \frac{\partial \mu^{s s}}{\partial p} .
$$

The first element $\partial x^{*} / \partial p$ in (27) and (28) is a static price effect that describes how consumption changes given $S$ and $\mu$. The overall response, however, includes a dynamic component, which on impact accounts for the adjustment in the policy function, given $S$, and in the long run also takes into account the effect of $p$ on the state variable.

With respect to the illustrative example of Section 2, this general model allows for considerably richer interactions, both in the utility function and in the law of motion, and produces short and long-run price effects that are in principle more complex. Yet, they admit a surprisingly compact representation, as shown below (all proofs are in the Appendix):

Proposition 1 (Price effect, no saving) At a steady state with saddle point stability of problem (12)-(14), a permanent increase in $p$ affects the consumption of $x$ as follows:

- On impact, the change in $x$ is

$$
\frac{\mathrm{d} x\left(S^{s s}\right)}{\mathrm{d} p}=\frac{\lambda^{s s}}{\Omega(\mathcal{H})|J(\mathcal{H})|} \varepsilon\left(f_{S}+f_{y} w_{S}-\rho\right)-x^{s s} \frac{\mathrm{d} x\left(S^{s s}\right)}{\mathrm{d} M}
$$

- In the long run, the change in steady state consumption of $x$ is

$$
\frac{\partial x^{s s}}{\partial p}=\frac{\lambda^{s s}}{\Omega(\mathcal{H})|J(\mathcal{H})|}\left(f_{S}+f_{y} w_{S}\right)\left(f_{S}+f_{y} w_{S}-\rho\right)-x^{s s} \frac{\partial x^{s s}}{\partial M}
$$

where $\Omega(\mathcal{H}),|J(\mathcal{H})|, \varepsilon, \frac{\partial x^{s s}}{\partial M}$ and $\frac{\mathrm{d} x\left(S^{s s}\right)}{\mathrm{d} M}$ are defined in equations (84), (85), (86), (95) and (99) in the Appendix, respectively.

Equations (29) and (30) characterize price effects in the short and in the long run for a dynamic consumer problem without saving. Similar to static price effects, also dynamic price effects can be distinguished into two components, both when considering the response 
on impact and in steady state. The first component is the dynamic counterpart of the substitution effect, represented by the first term in equations (29) and (30); the second component, represented by the terms $-x^{s s} \mathrm{~d} x\left(S^{s s}\right) / \mathrm{d} M$ and $-x^{s s} \partial x^{s s} / \partial M$, respectively, is the dynamic counterpart of the income effect.

Two novel results arise in an intertemporal setting. First, the law of demand can hold on impact, but not in steady state $\left(\mathrm{d} x\left(S^{s s}\right) / \mathrm{d} p<0\right.$ and $\left.\partial x^{s s} / \partial p>0\right)$, or instead be violated on impact but hold in steady state $\left(\mathrm{d} x\left(S^{s s}\right) / \mathrm{d} p>0\right.$ and $\left.\partial x^{s s} / \partial p<0\right)$. Second, both on impact and in steady state the dynamic counterpart of the substitution effect can be positive, and can therefore be by itself a source of violation of the law of demand. To focus on this new channel, the following Remark provides sufficient conditions under which there are no exogenous income effects:

Remark 2 (No income effects: sufficient conditions) In a stable steady state of problem (12)-(14), if the utility function is quasi linear in $y$, and $y$ does not affect the evolution of $S$, then there are no exogenous income effects: if $f_{y}=\mathcal{H}_{y S}=\mathcal{H}_{x y}=\mathcal{H}_{y y}=0$, then $\frac{\mathrm{d} x\left(S^{s s}\right)}{\mathrm{d} M}=\frac{\partial x^{s s}}{\partial M}=0$.

The conditions presented in Remark 2 are commonly assumed in the economic literature. They imply that $\lambda^{*}>0$ along the optimal path (and hence in steady state, see equation (17) above). Since $|J(\mathcal{H})|$, the determinant of the Jacobian matrix associated to (24)-(25), is negative in a steady state with saddle point stability, $\Omega(\mathcal{H})$ is positive by concavity and $\varepsilon$ is the negative eigenvalue of $J(\mathcal{H})$, the following holds:

Proposition 2 (Price effect absent income effect, no saving) Under the conditions of Remark 2, which rule out income effects, after a permanent increase in $p$ :

- If $f_{S}>0$ the consumption of $x$ responds non-monotonically over time:

$-x$ decreases on impact $\left(\frac{\mathrm{d} x\left(S^{s s}\right)}{\mathrm{d} p}<0\right)$ and increases in steady state $\left(\frac{\partial x^{s s}}{\partial p}>0\right)$ if $f_{S} \in(0, \rho)$,

$-x$ increases on impact $\left(\frac{\mathrm{d} x\left(S^{s s}\right)}{\mathrm{d} p}>0\right)$ and decreases in steady state $\left(\frac{\partial x^{s s}}{\partial p}<0\right)$ if $f_{S}>\rho ;$

- If $f_{S}<0$ :

- $x$ decreases both on impact and in steady state,

- $x$ is more elastic on impact than in steady state (hence it responds non-monotonically over time) if $f_{S}>\varepsilon$, that is if $f_{x}^{2} \mathcal{H}_{S S}+f_{x} \mathcal{H}_{x S}\left(\rho-2 f_{S}\right)<0$, and it is more rigid than in steady state (thus responding monotonically) otherwise. 
Absent income effects, the above Proposition emphasizes the role of time discounting and of the marginal effect of $S$ on its speed of change for dynamic price effects.

When $f_{S}$ is negative, the future consequences of current behavior are dampened. Condition $f_{S}<0$ is common in the economic literature, as it holds in models with a law of motion of the form $\dot{S}=g(x)-\delta S$. In such models, depreciation acts as a stabilizer because an initial increase in $S$ due to current choices raises depreciation and therefore slows down future growth of $S$. This adds to the effect of time discounting in making the future consequences of current actions less relevant. For this reason, when $f_{S}<0$ the dynamic behavior at any time horizon qualitatively resembles consumer behavior in a static set-up, where, absent income effects, demand falls in response to a price increase (see Appendix A.3). While the law of demand holds both on impact and in steady state, demand can have different price elasticity over different time horizons, according to the sign of $f_{x}^{2} \mathcal{H}_{S S}+f_{x} \mathcal{H}_{x S}\left(\rho-2 f_{S}\right)$. If this expression is positive, which occurs when $f_{x} \mathcal{H}_{x S}$ is positive and large, the response to a permanent price increase is monotonic and demand is more rigid on impact than in steady state. ${ }^{9}$ If instead the above expression is negative, demand on impact is more elastic than in the long run (i.e. there is overshooting in the short run) and the response of $x$ over time follows a non-monotonic path.

When $f_{S}$ is positive, instead, the future consequences of current behavior are amplified over time. ${ }^{10}$ This creates a trade-off between short and long run, to which consumers with different time preferences react in different ways. If $f_{S} \in(0, \rho)$, the amplification effect is small relative to the degree of impatience and the consumer favors the short over the long run, initially responding to a price increase as she would do in a static set-up, namely, in the absence of income effects, by reducing demand. In the long run, this initial choice has the costly consequence of leading to an increase in the steady state consumption of $x$, a consequence that is accepted precisely because long run effects are heavily discounted.

If instead $f_{S}>\rho$, the amplification effect is large relative to impatience and the consumer pays more attention to the long than to the short run. Accordingly, she is willing to accept an initial increase in consumption in response to a higher price, in order to follow a dynamic path leading to lower steady state consumption.

Notably, when $f_{S}$ is positive, the short and the long-run price effect have opposite sign and consumption of $x$ exhibits a non-monotonic time path in response to a rise in $p$ : either the law of demand holds on impact, and is violated in the long run, or the other way around. Hence a price-based policy can deter consumption of $x$ in the short run, but stimulate it

\footnotetext{
${ }^{9}$ This condition is met, for instance, in models of rational addiction, such as the one considered in Section 6.2 , where consumption of the addictive good increases addiction $\left(f_{x}>0\right)$ and endogenously reinforces the taste for the addictive good $\left(\mathcal{H}_{x S}>0\right)$, if the good is sufficiently addictive, i.e. if $\mathcal{H}_{x S}$ is large.

${ }^{10}$ Condition $f_{S}>0$ may hold, for instance, in models of human capital accumulation and renewable resources. The illustrative example displayed in Section 2 is a simple case in which $f_{S}=1$.
} 
in the long run, or the other way around.

In view of its relevance for many economic problems, and in particular for the related policy implications, we now consider the steady state response of the state variable to a change in price. $^{11}$

Proposition 3 (Price effect on $S^{s s}$, no saving) The steady state response of the state variable $S$ to a permanent increase in the market price $p$ of good $x$ is described by

$$
\frac{\partial S^{s s}}{\partial p}=\frac{\lambda^{s s}}{\Omega(\mathcal{H})|J(\mathcal{H})|}\left(p f_{y}-f_{x}\right)\left(f_{S}+f_{y} w_{S}-\rho\right)-x^{s s} \frac{\partial S^{s s}}{\partial M}
$$

where $\Omega(\mathcal{H}),|J(\mathcal{H})|$ and $\frac{\partial S^{s s}}{\partial M}$ are defined in equations (84), (85) and (93) in the Appendix, respectively.

In analogy to the previous analysis, we now present the steady state price response of $S$ when income effects are absent. ${ }^{12}$

Remark 3 (Price effect on $S^{s s}$ absent income effects, no saving) Under the conditions of Remark 2, the following holds:

- $\frac{\partial S^{s s}}{\partial p}=-\frac{f_{x}}{f_{S}} \frac{\partial x^{s s}}{\partial p}$

- $\frac{\partial S^{s s}}{\partial p}>0$ if and only if $f_{x}\left(f_{S}-\rho\right)>0$.

\section{A dynamic consumer problem with saving}

In this Section we extend the analysis to allow for the possibility of saving, lending and borrowing. We first consider the case in which the interest rate is endogenous, then we consider the case in which it is exogenously given.

\subsection{Endogenous interest rate}

When the agent can save or borrow, the static budget constraint considered in the previous Section is replaced by a dynamic budget constraint. Here we consider the case in which

\footnotetext{
${ }^{11}$ Equations (29), (30) and (31) can be related to expressions (18e), (12b) and (12a), respectively, in Caputo (1997). We differ from his analysis by explicitly considering price effects in a dynamic consumer problem and by also investigating, in the next Sections, problems with saving and time allocation.

${ }^{12}$ Under the conditions of Remark 2, also the steady state level of $S$ does not depend on exogenous income $\left(\partial S^{s s} / \partial M=0\right)$, see equation (93) in the Appendix.
} 
returns on assets $r(A)$ are increasing and strictly concave. The dynamic consumer problem now is

$$
\begin{aligned}
\max _{\{x, y\}} & \int_{0}^{\infty} e^{-\rho t} U(x, y, S) \mathrm{d} t \\
\text { s.t. } & \dot{A}=r(A)+M+w(S)-p x-y \\
& \dot{S}=f(x, y, S)
\end{aligned}
$$

with $S(0)=S_{0}, A(0)=A_{0}, x(t) \geq 0$ and $y(t) \geq 0$. The corresponding current-value Hamiltonian function is:

$$
\tilde{\mathcal{H}}(x, y, S, A, \mu, \lambda ; p, M)=U(x, y, S)+\lambda[r(A)+M+w(S)-p x-y]+\mu f(x, y, S)
$$

where $\lambda$ and $\mu$ are the costate variables associated to the states $A$ and $S$, respectively. ${ }^{13}$ The following conditions are necessary for an internal solution:

$$
\begin{aligned}
\tilde{\mathcal{H}}_{x} & =U_{x}(x, y, S)-\lambda p+\mu f_{x}(x, y, S)=0 \\
\tilde{\mathcal{H}}_{y} & =U_{y}(x, y, S)-\lambda+\mu f_{y}(x, y, S)=0 \\
\dot{\lambda} & =\lambda\left(\rho-r_{A}\right) \\
\dot{\mu} & =\rho \mu-\tilde{\mathcal{H}}_{S}(x, y, S, A, \mu, \lambda ; p, M) \\
\dot{A} & =r(A)+M+w(S)-p x-y \\
\dot{S} & =f(x, y, S)
\end{aligned}
$$

with transversality conditions $\lim _{t \rightarrow \infty} e^{-\rho t} \mu(t) S(t)=0$ and $\lim _{t \rightarrow \infty} e^{-\rho t} \lambda(t) A(t)=0$. The above conditions are also sufficient for a maximum if $\tilde{\mathcal{H}}(x, y, S, A, \mu, \lambda ; p, M)$ is concave in the state and control variables (Mangasarian, 1966; Seierstad and Sydsaeter, 1977). We additionally assume $\tilde{\mathcal{H}}_{x x}$ and $\tilde{\mathcal{H}}_{y y}$ to be strictly negative.

Proceeding as in the previous Section allows obtaining the steady state consumption of $x$ and the corresponding policy function:

$$
\begin{aligned}
& x^{s s}=x^{s s}(p, M)=x^{*}\left(S^{s s}, A^{s s}, \mu^{s s}, \lambda^{s s} ; p, M\right) \\
& x(S, A)=x^{*}(S, A, \mu(S, A), \lambda(S, A) ; p, M)
\end{aligned}
$$

where $x^{*}$ represents the value of $x$ that satisfies the first order conditions (36) and (37).

\footnotetext{
${ }^{13}$ In the previous Section $\lambda$ was the Lagrange multiplier associated to the static budget constraint. Here, with a slight abuse of notation, it represents the costate variable associated to the dynamic budget constraint. We focus on the economically relevant case in which the shadow value of wealth $\lambda$ is positive along the optimal path.
} 
The following holds:

Proposition 4 (Price effect with saving) At a steady state with saddle point stability of problem (32)-(34), a permanent increase in $p$ affects the consumption of $x$ as follows:

- On impact, the change in $x$ is

$$
\frac{\mathrm{d} x\left(S^{s s}, A^{s s}\right)}{\mathrm{d} p}=\frac{\left(\lambda^{s s}\right)^{2} r_{A A}}{\tilde{\Omega}|\tilde{J}|} \tilde{\varepsilon}\left(f_{S}+f_{y} w_{S}-\rho\right)-x^{s s} \frac{\mathrm{d} x\left(S^{s s}, A^{s s}\right)}{\mathrm{d} M} ;
$$

- In the long run, the change in steady state consumption of $x$ is

$$
\frac{\partial x^{s s}}{\partial p}=\frac{\left(\lambda^{s s}\right)^{2} r_{A A}}{\tilde{\Omega}|\tilde{J}|}\left(f_{S}+f_{y} w_{S}\right)\left(f_{S}+f_{y} w_{S}-\rho\right)-x^{s s} \frac{\partial x^{s s}}{\partial M}
$$

where $|\tilde{J}|, \tilde{\Omega}, \frac{\partial x^{s s}}{\partial M}, \frac{\mathrm{d} x\left(S^{s s}, A^{s s}\right)}{\mathrm{d} M}$ and $\tilde{\varepsilon}$ are defined in equations (112), (113), (129), (137) and (138) in the Appendix, respectively.

Proposition 4 shows that price effects in a dynamic consumer's problem with saving at an endogenous interest rate closely parallel the price effects presented in Proposition 1 for the problem without saving, except for the fact that all terms are now derived from a different Hamiltonian function, that saddle point stability requires $|\tilde{J}|>0$, and that there is an additional term $\lambda^{s s} r_{A A}$ to account for the dynamic budget constraint. Hence, even in presence of borrowing and lending, price effects can still be decomposed into an exogenous income effect and a dynamic analogue of the substitution effect. They can also produce monotonic and non-monotonic patterns of consumption, price responses that have different sign over different time horizons, and possible violations of the law of demand at some point in time, as it is clearly shown in the case considered below. ${ }^{14}$

Proposition 5 (Price effect in a simple case with saving) In a stable steady state of problem (32)-(34), if $f_{y}=\tilde{\mathcal{H}}_{y S}=\tilde{\mathcal{H}}_{x y}=w_{S}=0$ and $\tilde{\varepsilon}<0$, then after a permanent increase in the market price $p$ :

- If $f_{S}>0$ the consumption of $x$ responds non-monotonically over time:

\footnotetext{
${ }^{14}$ For the problem without saving, in Remark 2 we stated that $f_{y}=\mathcal{H}_{y S}=\mathcal{H}_{x y}=\mathcal{H}_{y y}=0$ are sufficient conditions for the absence of income effects. In the current problem this set of conditions is not admissible because strict concavity in both controls precludes $\tilde{\mathcal{H}}_{y y}=0$ (and implies $\tilde{\Omega}>0$ ). Replacing $w_{S}=0$ for $\tilde{\mathcal{H}}_{y y}=0$ we obtain a simple case in which income effects are non nil. Proposition 5 adds condition $\tilde{\varepsilon}<0$, which always holds in our numerical simulations.
} 
$-x$ decreases on impact $\left(\frac{\mathrm{d} x\left(S^{s s}, A^{s s}\right)}{\mathrm{d} p}<0\right)$ and increases in steady state $\left(\frac{\partial x^{s s}}{\partial p}>0\right)$ if $f_{S} \in(0, \rho)$,

- $x$ increases on impact $\left(\frac{\mathrm{d} x\left(S^{s s}, A^{s s}\right)}{\mathrm{d} p}>0\right)$ and decreases in steady state $\left(\frac{\partial x^{s s}}{\partial p}<0\right)$ if $f_{S}>\rho$;

- If $f_{S}<0$ :

- $x$ decreases both on impact an in steady state,

- $x$ is more elastic on impact than in steady state (hence it responds non-monotonically over time) if $f_{S}>\tilde{\varepsilon}$, and it is more rigid than in steady state (thus responding monotonically) otherwise.

Let us now consider the long run response of the state variable $S$ to price changes:

Proposition 6 (Price effect on $S^{s s}$ with saving) The steady state response of the state variable $S$ to a permanent increase in the market price $p$ of good $x$ is described by

$$
\frac{\partial S^{s s}}{\partial p}=\frac{\left(\lambda^{s s}\right)^{2} r_{A A}}{\tilde{\Omega}|\tilde{J}|}\left(p f_{y}-f_{x}\right)\left(f_{S}+f_{y} w_{S}-\rho\right)-x^{s s} \frac{\partial S^{s s}}{\partial M}
$$

where $|\tilde{J}|, \tilde{\Omega}$, and $\frac{\partial S^{s s}}{\partial M}$ are defined in equations (112), (113) and (124) in the Appendix, respectively.

In the simple case, the response of $S$ to a price change satisfies the properties described below: ${ }^{15}$

Remark 4 (Price effect on $S^{s s}$ in a simple case with saving) In a stable steady state of problem (32)-(34), if $f_{y}=\tilde{\mathcal{H}}_{y S}=\tilde{\mathcal{H}}_{x y}=w_{S}=0$, the following holds:

- $\frac{\partial S^{s s}}{\partial p}=-\frac{f_{x}}{f_{S}} \frac{\partial x^{s s}}{\partial p}$

- $\frac{\partial S^{s s}}{\partial p}>0$ if and only if $f_{x}\left(f_{S}-\rho\right)>0$.

\footnotetext{
${ }^{15}$ In the simple case, the steady state value of $S$ increases with exogenous income $\left(\partial S^{s s} / \partial M>0\right)$ if and only if $f_{x}\left(f_{S}-\rho\right)<0$, see equation (124).
} 


\subsection{Exogenous interest rate}

After considering the case of saving with decreasing marginal returns to assets, an assumption that is reasonable and common in macroeconomic modeling, we now consider the microeconomic perspective in which individual consumers take the market interest rate as given. In such a case, the literature often focuses on linear asset returns and studies Frisch demand functions, in which price changes are compensated to keep the shadow value of assets constant. Following this literature, we now assume that asset income is $r(A)=r A$, where $r=\rho>0$ (see, e.g., Heckman, 1974; Becker and Murphy, 1988). Under these conditions, equation (38) implies that the costate variable $\lambda$ associated to the dynamic budget constraint is constant, hence the dynamic system reduces to equations (39)-(41), once the FOCs (36)-(37) are satisfied. Since expression (43) is now independent of $A$ (see Appendix), we can write the policy function simply as $x(S)$ and state the following:

Proposition 7 (Price effect for Frisch demand functions) At a steady state with saddle point stability of problem (32)-(34), with $r(A)=r A$ and $r=\rho$, a permanent increase in $p$ that is compensated to maintain the shadow value of assets $\lambda_{0}$ constant affects the consumption of $x$ as follows:

- On impact, the change in $x$ is:

$$
\begin{aligned}
\frac{\mathrm{d} x\left(S^{s s}\right)}{\mathrm{d} p}= & \frac{\lambda_{0} \rho}{\Phi \tilde{\Omega}\left|J_{0}\right|} \varepsilon_{0}\left(f_{S}-\rho\right)\left(f_{y} \tilde{\mathcal{H}}_{x y}-f_{x} \tilde{\mathcal{H}}_{y y}\right)^{2} \\
& +\frac{\lambda_{0} f_{y}}{\Phi}\left[\frac{\rho}{\tilde{\Omega}\left|J_{0}\right|} \varepsilon_{0}\left(f_{y} \tilde{\mathcal{H}}_{x y}-f_{x} \tilde{\mathcal{H}}_{y y}\right)\left(f_{x} \tilde{\mathcal{H}}_{y S}-f_{y} \tilde{\mathcal{H}}_{x S}\right)-f_{y}\right]
\end{aligned}
$$

- In the long run, the change in steady state consumption of $x$ is

$$
\frac{\partial x^{s s}}{\partial p}=-\frac{\rho \lambda_{0}}{\tilde{\Omega}\left|J_{0}\right|}\left\{f_{S}\left(f_{S}-\rho\right) \tilde{\mathcal{H}}_{y y}+f_{y}\left[f_{y} \tilde{\mathcal{H}}_{S S}+\left(\rho-2 f_{S}\right) \tilde{\mathcal{H}}_{y S}\right]\right\}
$$

where $\tilde{\Omega},\left|J_{0}\right|, \varepsilon_{0}$, and $\Phi$ are defined in equations (113), (154), (164) and (166) in the Appendix, respectively.

A main difference between Proposition 7 and Propositions 1 and 4 is that, for Frisch demand functions, the income effect is nil both on impact and in steady state $\left(\mathrm{d} x\left(S^{s s}\right) / \mathrm{d} M=0\right.$ and $\left.\partial x^{s s} / \partial M=0\right)$. The price effects in equations (47) and (48) are therefore only due to intertemporal substitution. Such intertemporal substitution effect is however different from the ones computed in the previous models because it considers a compensation that keeps the marginal utility of assets constant. 
Since $\lambda_{0}>0, \Phi>0$, and since $\tilde{\Omega}$ is positive by concavity and $\left|J_{0}\right|$ is negative in a steady state with saddle point stability (now the Jacobian matrix is 3-dimensional and has at most one negative eigenvalue), in the special case in which the dynamics of state $S$ is not affected by control $y$ one obtains:

Proposition 8 (Price effect for Frisch demand functions when $\mathbf{f}_{\mathbf{y}}=\mathbf{0}$ ) In a steady state with saddle point stability of problem (32)-(34), with $r(A)=r A, r=\rho$ and $f_{y}=0$, after a permanent increase in the market price $p$ :

- If $f_{S}>0$ the consumption of $x$ responds non-monotonically over time:

$-x$ decreases on impact $\left(\frac{\mathrm{d} x\left(S^{s s}\right)}{\mathrm{d} p}<0\right)$ and increases in steady state $\left(\frac{\partial x^{s s}}{\partial p}>0\right)$ if $f_{S} \in(0, \rho)$,

$-x$ increases on impact $\left(\frac{\mathrm{d} x\left(S^{s s}\right)}{\mathrm{d} p}>0\right)$ and decreases in steady state $\left(\frac{\partial x^{s s}}{\partial p}<0\right)$ if $f_{S}>\rho ;$

- If $f_{S}<0$ :

- $x$ decreases both on impact an in steady state,

- $x$ is more elastic on impact than in steady state (hence it responds non-monotonically over time) if $f_{S}>\varepsilon_{0}$, and it is more rigid than in steady state (thus responding monotonically) otherwise.

We thus have that, when $y$ does not affect the evolution of the state variable $S$, the same simple conditions on $f_{S}$ and $\rho$ already obtained in the absence of income effects in Proposition 2, and in the simple case with endogenous interest rate in Proposition 5, characterize short and long-run (Frisch) price effects, and they can produce non-monotonic consumption paths in response to price changes, price effects of different sign over different time horizons and violations of the law of demand at some point in time. ${ }^{16}$

Let us now consider the long run response of the state variable $S$ to price changes:

Proposition 9 (Price effect on $S^{s s}$ for Frisch demand functions) The steady state response of the state variable $S$ to a permanent increase in the market price $p$ of good $x$ is described by

$$
\frac{\partial S^{s s}}{\partial p}=\frac{\rho \lambda_{0}}{\tilde{\Omega}\left|J_{0}\right|}\left\{f_{x}\left(f_{S}-\rho\right) \tilde{\mathcal{H}}_{y y}+f_{y}\left[f_{y} \tilde{\mathcal{H}}_{x S}-\left(f_{S}-\rho\right) \tilde{\mathcal{H}}_{x y}-f_{x} \tilde{\mathcal{H}}_{y S}\right]\right\}
$$

\footnotetext{
${ }^{16}$ If the dynamics of state $S$ is also affected by control $y$, another possible source of violations comes from the term $f_{y}\left(\rho-2 f_{S}\right) \tilde{\mathcal{H}}_{y S}$ (notice that the term $f_{y}^{2} \tilde{\mathcal{H}}_{S S}$ cannot be positive). As we know from the static theory of Giffen goods, and as already discussed above, interactions in the objective function can be a source of violation of the law of demand. What is interesting to notice here is that the relevant interaction is now between state $S$ and control $y$.
} 
where $\tilde{\Omega}$ and $\left|J_{0}\right|$ are defined in equations (113) and (154) in the Appendix, respectively.

When $f_{y}=0$, the response of $S$ to a change in $p$ satisfies the following properties:

Remark 5 (Price effect on $S^{s s}$ for Frisch demand functions when $\mathbf{f}_{\mathbf{y}}=\mathbf{0}$ ) In a stable steady state of problem (32)-(34), with $r(A)=r A$ and $r=\rho$, if $f_{y}=0$, then

- $\frac{\partial S^{s s}}{\partial p}=-\frac{f_{x}}{f_{S}} \frac{\partial x^{s s}}{\partial p}$,

- $\frac{\partial S^{s s}}{\partial p}>0$ if and only if $f_{x}\left(f_{S}-\rho\right)>0$.

\section{A dynamic labor supply problem}

In this Section we consider an important class of intertemporal consumer problems, involving the choice between consumption and time allocation to labor and other activities. To study how labor supply responds over time to wage changes we use a slight modification of the problem with saving and exogenous interest rate just discussed in Section 4.2.

Let $x$ be the share of time devoted to non-labor activities (leisure, training or other activities, depending on the application of interest), and $y$ be consumption. The natural counterpart of price effects are now wage effects. We will characterize the short and long-run response of Frisch labor supply in the following individual problem:

$$
\begin{aligned}
\max _{\{x, y\}} & \int_{0}^{\infty} e^{-\rho t} U(x, y, S) \mathrm{d} t \\
\text { s.t. } & \dot{S}=f(x, y, S) \\
& \dot{A}=r A+M+w g(x, S)-y,
\end{aligned}
$$

where $g(x, S)$ represents effective labor and $w$ is its exogenous return. To accommodate for a variety of models that are commonly used in the economic literature, including human capital, health, addiction and natural resources, we assume $g(x, S)$ to be continuously differentiable and decreasing in non-labor time, $g_{x}<0$, but we make no specific assumption on the effect of state $S$ on effective labor. The main formal difference with respect to the previous Section is that now state $S$ and control $x$ do not enter the dynamic budget constraint in an additively separable way, but can potentially interact. We also make no specific assumptions on how instantaneous utility depends on $S$. The time endowment is normalized to one, so that the share of labor time is $\ell=1-x$.

The current-value Hamiltonian is

$$
\check{\mathcal{H}}(x, y, S, A, \mu, \lambda ; w, M)=U(x, y, S)+\lambda[r A+M+w g(x, S)-y]+\mu f(x, y, S)
$$


which is assumed to be concave in state and control variables, with $\check{\mathcal{H}}_{x x}<0$ and $\check{\mathcal{H}}_{y y}<0$. Proceeding as in the previous Sections one obtains:

Proposition 10 (Wage effect for Frisch labor supply) In a steady state with saddle point stability of problem (50)-(52), with $r(A)=r A$ and $r=\rho$, a permanent increase in the wage $w$ paid per unit of effective labor that is compensated to maintain the shadow value of assets $\lambda_{0}$ constant affects Frisch labor supply $\ell$ as follows:

- On impact, the change in $\ell$ is:

$$
\begin{aligned}
\frac{\mathrm{d} \ell\left(S^{s s}\right)}{\mathrm{d} w}= & -\frac{\lambda_{0}}{\check{\Phi}} f_{y}^{2} g_{x}+\frac{\rho \lambda_{0}}{\check{\Omega}|\check{J}|} \check{\varepsilon}\left(f_{x} \check{\mathcal{H}}_{y y}-f_{y} \check{\mathcal{H}}_{x y}\right)\left[g_{S}\right. \\
& \left.+g_{x} \frac{\left(f_{x} \check{\mathcal{H}}_{y y}-f_{y} \check{\mathcal{H}}_{x y}\right)\left(f_{S}-\rho\right)+f_{y}\left(f_{y} \check{\mathcal{H}}_{x S}-f_{x} \check{\mathcal{H}}_{y S}\right)}{\check{\Phi}}\right] ;
\end{aligned}
$$

- In the long run, the change in steady state labor supply $\ell$ is:

$$
\begin{aligned}
\frac{\partial \ell^{s s}}{\partial w}= & \frac{\rho \lambda_{0}}{\check{\Omega}|\check{J}|} g_{S}\left[f_{S} f_{x} \check{\mathcal{H}}_{y y}+f_{y}\left(f_{y} \check{\mathcal{H}}_{x S}-f_{S} \check{\mathcal{H}}_{x y}-f_{x} \check{\mathcal{H}}_{y S}\right)\right] \\
& -\frac{\rho \lambda_{0}}{\check{\Omega}|\check{J}|} g_{x}\left\{f_{S}\left(f_{S}-\rho\right) \check{\mathcal{H}}_{y y}+f_{y}\left[f_{y} \check{\mathcal{H}}_{S S}+\left(\rho-2 f_{S}\right) \check{\mathcal{H}}_{y S}\right]\right\}
\end{aligned}
$$

where $\check{\Phi}, \check{\Omega},|\check{J}|$ and $\check{\varepsilon}$ are defined in equations (178), (179), (180) and (181) in the Appendix, respectively.

Proposition 10 shows that, in a dynamic problem, labor supply can respond non-monotonically to wage changes and that, in general, the sign of the response to wage is ambiguous, despite income effects being nil both on impact and in steady state $\left(\mathrm{d} \ell\left(S^{s s}\right) / \mathrm{d} M=\partial \ell^{s s} / \partial M=\right.$ $0) .{ }^{17}$ This can be immediately appreciated when state $S$ is not influenced by consumption $y$. In such a case, given $\lambda_{0}>0$, and since $\check{\Omega}$ and $\check{\Phi}$ are positive by concavity, and $|\check{J}|$ and $\check{\varepsilon}$ are negative in a steady state with saddle point stability, the following holds:

Proposition 11 (Wage effect for Frisch labor supply when $\mathbf{f}_{\mathbf{y}}=\mathbf{0}$ ) In a steady state with saddle point stability of problem (32)-(34), with $r(A)=r A, r=\rho$ and $f_{y}=0$, after a permanent increase in the wage $w$ :

- If $f_{S}>0$ labor supply $\ell$ responds non-monotonically over time:

\footnotetext{
${ }^{17}$ If effective labor does not depend on state $S$ (i.e. $g_{S}=0$ ) and $g_{x}=-1$, then, mutatis mutandis, expressions (54) and (55) reduce to (47) and (48), obviously with the opposite sign since we are now looking at labor supply rather than at leisure (or training) demand.
} 
$-\ell$ increases on impact $\left(\frac{\mathrm{d} \ell\left(S^{s s}\right)}{\mathrm{d} w}>0\right)$ and decreases in steady state $\left(\frac{\partial \ell^{s s}}{\partial w}<0\right)$ if $f_{S}<\check{\rho}$,

$-\ell$ decreases on impact $\left(\frac{\mathrm{d} \ell\left(S^{s s}\right)}{\mathrm{d} w}<0\right)$ and increases in steady state $\left(\frac{\partial \ell^{s s}}{\partial w}>0\right)$ if $f_{S}>\check{\rho}$

- If $f_{S}<0$ :

- $\ell$ increases both on impact an in steady state if $f_{S}<\check{\rho}$, and it decreases both on impact an in steady state if $f_{S}>\check{\rho}$;

- $\ell$ is more elastic on impact than in steady state (hence it responds non-monotonically over time) if $f_{S}>\check{\varepsilon}$, and it is more rigid than in steady state (thus responding monotonically) otherwise

where $\check{\rho}=\rho+g_{S} f_{x} / g_{x}$.

Once again, despite the complexity of an intertemporal model of labor supply, the short and long-run response of labor supply to wage changes can depend on surprisingly compact conditions. Consistent with the results presented in Proposition 8, if $f_{S}>0$, labor supply responds non-monotonically over time and the sign of the short-run reaction is opposite to the long-run response. Labor supply increases on impact but decreases in the long run, or the other way around, depending on whether $f_{S}$ is smaller or larger than $\check{\rho}$, respectively. Since the threshold value $\check{\rho}$ increases with impatience $\rho$, the intuition behind this result is similar to the one proposed in the previous Sections, with the only difference that now $\check{\rho}$ also incorporates the role of effective labor. ${ }^{18}$

If instead $f_{S}<0$, the short and long-run wage responses of labor supply have the same sign. In particular, if impatience is large $\left(f_{S}<\check{\rho}\right)$, the qualitative reaction parallels the one obtained in a static labor supply model, in which, absent income effects, an increase in the opportunity cost of leisure induces to work more. If instead the agent is patient $\left(f_{S} \in(\check{\rho}, 0)\right)$, an increase in the returns to effective labor induces the agent to work less, both in the short and in the long run. As in the previous Sections, when $f_{S}<0$ it remains true that the monotonicity of the time path of the wage response of labor supply depends on whether $f_{S}>\check{\varepsilon}$ or the other way around.

In the context of the models discussed in Section 6.1, Proposition 11 implies that labor supply increases both in the short and in the long run if human capital is accumulated through learning-by-doing. If instead human capital is accumulated through training, patient agents can respond to higher returns to effective labor by working less and investing

\footnotetext{
${ }^{18}$ Note that $\check{\rho}$ can be either positive or negative. If $g_{S}=0$, then $\check{\rho}=\rho$ and the wage effect depends on $f_{S} \lessgtr \rho$, as the price effect in Proposition 8 .
} 
more in training. In the absence of income effects, this negative response of labor supply to a wage increase runs against the predictions based on the static substitution effect: now the agent chooses to dynamically raise effective labor supply through more training and higher human capital, rather than by working more.

As done in the previous Sections, the long run response of the state variable $S$ to a wage change can be explicitly assessed:

Proposition 12 (Wage effect on $S^{s s}$ for Frisch labor supply) The steady state response of the state variable $S$ to a permanent increase in wage $w$ is described by

$$
\frac{\partial S^{s s}}{\partial w}=-\frac{\rho \lambda_{0}}{\check{\Omega}|\check{J}|}\left\{g_{S} \check{\Phi}+g_{x}\left[\left(f_{S}-\rho\right)\left(f_{x} \check{\mathcal{H}}_{y y}-f_{y} \check{\mathcal{H}}_{x y}\right)+f_{y}^{2} \check{\mathcal{H}}_{x S}-f_{x} f_{y} \check{\mathcal{H}}_{y S}\right]\right\}
$$

When $f_{y}=0$, the response to a wage change satisfies the following properties:

Remark 6 (Wage effect on $S^{s s}$ for Frisch labor supply when $\mathbf{f}_{\mathbf{y}}=\mathbf{0}$ ) At a stable steady state of problem (50)-(52), with $r(A)=r A$ and $r=\rho$, if $f_{y}=0$, then

- $\frac{\partial S^{s s}}{\partial w}=\frac{f_{x}}{f_{S}} \frac{\partial \ell^{s s}}{\partial w}$,

- $\frac{\partial S^{s s}}{\partial w}>0$ if and only if either $f_{x}>0$ and $f_{S}>\check{\rho}$, or $f_{x}<0$ and $f_{S}<\check{\rho}$.

\section{Applications}

The dynamic consumer problems analyzed in the previous Sections encompass a number of models commonly used in the economic literature. To appreciate it, in this Section we consider classic workhorse models of human capital accumulation, addiction, habit and taste formation, health, and exploitation of natural resources. This selection illustrates the wide applicability of our results to a variety of fields in economic research, but is clearly not exhaustive. ${ }^{19}$

\subsection{Human capital accumulation}

Models of human capital accumulation can be distinguished in two broad classes: training models, which focus on the direct investment in human capital accumulation through either schooling or on-the-job training (see, e.g., Ben-Porath, 1967; Heckman, 1976; Blinder and Weiss, 1976), and learning by doing (or experience) models, which consider human capital

\footnotetext{
${ }^{19}$ For each application we have verified through numerical simulations that there is a non-empty parameter set such that a stable steady state exists, concavity and non-negativity assumptions are satisfied, and the conditions identifying short and long run responses in each case hold.
} 
accumulation as a byproduct of work activity (see, e.g., Arrow, 1962; Weiss, 1972). The essence of both classes is captured by model (50)-(52), where $S$ is human capital, effective labor increases in $S\left(g_{S}>0\right)$, and $y$ is a consumption good. A common assumption is that $y$ does not affect human capital accumulation $\left(f_{y}=0\right)$ and that human capital does not affect instantaneous utility $\left(U_{S}=0\right)$. The main difference between the two classes of models is that $x$ is either interpreted as a training investment, which contributes to human capital accumulation, or as leisure, which yields utility but reduces labor time and consequently learning by doing. ${ }^{20}$ Thus in training models $f_{x}>0$, whereas in learning models $f_{x}<0 .{ }^{21}$

Let us set $r=\rho$ for simplicity and focus on Frisch supply function, which rules out income effects. Since consumption $y$ does not affect human capital accumulation, Proposition 11 and Remark 6 apply and allow identifying short and long run responses of labor supply to a wage increase, as well as predicting the evolution of human capital over time, both for training and for learning models.

Let us focus on $\check{\rho}>0$, a condition that always holds in learning models and also holds in training models for sufficiently impatient agents (recall that $\check{\rho}=\rho+f_{x} g_{S} / g_{x}$ ). If in steady state $f_{S}>0$, labor supply responds to a wage increase with opposite sign in the short and in the long run. Such response is heterogeneous and depends on the agent's degree of impatience. Impatient workers $\left(f_{S} \in(0, \check{\rho})\right)$ initially increase and then decrease their labor supply. The initial increase in fact mimics the standard response one would expect in a static framework where, absent income effects, substitution effects drive the reaction to a wage increase. When considering training models, the higher initial labor supply leaves less time for training, so that human capital and productivity start decreasing. Given that $f_{S}>0$, this initial fall in human capital feeds back and amplifies over time. To prevent this from happening, an impatient worker progressively increases the time devoted to training, eventually reaching a new steady state in which both labor supply and human capital are lower than in the old steady state, as the higher subsequent training investment only partially compensates for the initial fall in human capital. When considering learning models, by contrast, the short run increase in labor supply leads a progressive accumulation

\footnotetext{
${ }^{20} \mathrm{~A}$ minor difference, immaterial for our results, is that instantaneous utility increases in leisure but decreases in training investment.

${ }^{21}$ In the literature, there are two main aspects in which human capital accumulation models may differ from the present setup: existing models may consider a finite time horizon, and they may allow for more than two control variables. On the one hand, an infinite time horizon model can represent a setting in which life is finite, but its duration is uncertain, along the lines of Yaari (1965). On the other hand, the model with two control variables captures in the simplest way the trade-off in the allocation of time between labor and either training or leisure. Killingsworth (1982) shows how training and learning can be combined into a broader model. It is easy to extend the analysis to the case of three control variables (consumption and the allocation of time among leisure, labor and training). A full analysis of finite-horizon life cycle models is also possible, but is beyond the scope of this article.
} 
of human capital. Again, with $f_{S}>0$, this initial change in human capital feeds back and amplifies over time as it leads to faster learning. Higher productivity allows the worker to subsequently reduce labor supply until reaching a new steady state where labor supply is lower than in the old one, but human capital is higher. Patient workers $\left(f_{S}>\check{\rho}\right)$ display exactly the opposite pattern.

If instead $f_{S}<0$, then after a permanent rise in wage both short and long run labor supply increase. If $f_{S}<\check{\varepsilon}$, labor supply jumps up on impact and keeps increasing over time. This monotonic response is due to labor supply being more rigid in the short than in the long run. If instead $f_{S}>\check{\varepsilon}$, labor supply is more elastic in the short than in the long run. This results in an initial overshooting relative to the long run labor supply increase, and in a non-monotonic path. As above, this is true in both training and learning models, with the difference that in the former human capital decreases, while in the latter it increases.

\subsection{Addiction, habit and taste formation}

Becker and Murphy (1988) develop a rational addiction model in which the consumer chooses between an addictive good $x$ and a non-addictive good $y$. Consumption of the addictive good contributes to the accumulation of addiction capital $S$, which in turn makes $x$ more desirable $\left(U_{x S}>0\right)$. Their model is a special case of model (32) to (34), in which $f(x, y, S)=x-\delta S$, the utility function is quadratic, $M=0$, and $r=\rho$. Since $f_{y}=0$ and $f_{S}=-\delta<0$, according to Proposition 8 and Remark 5, in response to a price increase consumption of the addictive good decreases both on impact and in steady state, leading to a lower level of addiction.

Becker and Murphy mention, but do not formally develop, the possibility of an investment in addiction reduction (for instance, participation in a rehabilitation program). Suppose $y$ is interpreted as such an investment and for simplicity assume $f(x, y, S)=x-y-\delta S$. One can imagine that participating to the rehab program not only involves expenditures, but is also physically or mentally costly, and that it is more costly the higher the level of addiction, that is $U_{y}<0$ and $U_{y S}<0$. According to Proposition 7, if $U_{y S}$ is sufficiently large in absolute value (and the marginal utility of wealth is positive), then an increase in the price of the addictive good can lead to a long-run increase in its consumption (possibly through a non monotonic path). ${ }^{22}$ It can be shown that the steady state rise in consumption of the addictive good $x$ is accompanied by an increase in rehab expenditure $y$, so that the overall effect is a lower steady state stock of addiction $S .{ }^{23}$ A higher price of the

\footnotetext{
${ }^{22}$ Formally, $\partial x^{s s} / \partial p>0$ if $\lambda_{0}>0$ and $U_{y S}<\left[\delta(\delta+\rho) U_{y y}+U_{S S}+\lambda_{0} w_{S S}\right] /(2 \delta+\rho)<0$.

${ }^{23}$ By combining Propositions 7 and 9 , the condition for steady state stability and the expression for $\partial y^{s s} / \partial p$, which can be computed in analogy to $\partial x^{s s} / \partial p$, one finds that in a stable steady state $\partial x^{s s} / \partial p>0$ implies $\partial y^{s s} / \partial p>0$ and $\partial S^{s s} / \partial p<0$.
} 
addictive good (e.g., higher taxes on alcohol) may thus eventually reduce addiction but, paradoxically, through an increase in the consumption of both alcohol and rehab services.

Alternatively, consider a variation of the model in which $y$ is also addictive (i.e. $U_{y S}>0$ ) and contributes to the addiction stock $S$ (e.g., $x$ is beer and $y$ is vodka). According to Proposition 7 , in a stable steady state the consumption of $x$ increases in its price when good $y$ is sufficiently more addictive than good $x .^{24}$ In this case, responding to an increase in the price of $x$ by substituting $y$ for $x$ would excessively increase addiction. To avoid it, the consumer reduces $y$ and increases $x$ (as the latter is less addictive) and ultimately the stock of addiction decreases. A higher tax on beer may thus reduce alcoholism but, paradoxically, through a reduction in vodka consumption and an increase in beer consumption.

With appropriate adaptations, models such as (12)-(14) in the absence of savings, or (32)-(34) when there is a credit market, can also capture taste formation or habit formation along the lines proposed by Stigler and Becker (1977) and by Abel (1990) and Carroll et al. (2000), respectively. In such situations consumption of good $x$ contributes to the accumulation of a specific consumption capital $S$, which feeds back into the demand for that good. The main difference is that consumption capital raises the marginal utility of $x$ in taste formation models $\left(U_{x S}>0\right.$ : the more I listen to music the more I appreciate it), while it reduces it in habit formation models $\left(U_{x S}<0\right.$ : the more I get used to a good, the less I value it). However, if the specific consumption capital does not affect income $\left(w_{S}=0\right)$ and is only accumulated through $x\left(f_{y}=0\right)$, and if the utility function is additively separable in $y$, then this difference is not relevant for the sign of the steady state price effect and either Proposition 2, or 5 or 8 , depending on the specification, applies. In particular, if in steady state $f_{S}>0$, then the consumption of $x$ responds in opposite ways to a price increase in the short and in the long run.

\subsection{Health capital}

Grossman (1972) proposes a health accumulation model in discrete-time. Its essence is captured by the continuous-time model (32)-(34), where $S$ is health capital, $x$ is a health investment (say, medical care) and $y$ is a consumption good, with $U(x, y, S)=V(y, S)$ and $f(x, y, S)=h(x)-\delta S, M=0$, where $h(\cdot)$ is an increasing and concave function and $r=\rho$. Since $f_{y}=0$ and $f_{S}=-\delta<0$, according to Proposition 8 the investment in health satisfies the law of demand both on impact and in steady state.

Consider now a variation of the model in which the consumer can increase health through medical care $x$ and physical activity $y$. For simplicity, consider $f(x, y, S)=h(x)+$

\footnotetext{
${ }^{24}$ Formally, $\partial x^{s s} / \partial p>0$ if $\lambda_{0}>0$ and $U_{y S}>-\left[\delta(\delta+\rho) U_{y y}+U_{S S}+\lambda_{0} w_{S S}\right] /(2 \delta+\rho)>0$, which in a stable steady state implies $U_{y S}>k U_{x S}$, where $k>1$.
} 
$y-\delta S$. It is natural to assume that physical activity is more enjoyable (or less costly) for healthier people, that is $U_{y S}>0$. This effect has the potential to lead to violations of the law of demand in the long run: when it is sufficiently strong, according to Proposition 7 , in a stable steady state an increase in the price of medical care leads to an increase in the consumption of medical care. ${ }^{25}$ It can also be shown that this is accompanied by a decrease in physical activity and a decrease in health. A higher price of medical care may thus reduce health but, surprisingly, through a reduction in physical exercise and an increase in the consumption of medical care.

\subsection{Natural resources}

In the tradition of Clark et al. (1979), environmental economics has devoted substantial attention to the problem of optimal exploitation of renewable resources. Depending on the application of interest, these problems may be considered from the point of view of a social planner, an extracting firm, or an individual. In the current framework it is natural to consider the last perspective.

Consider a model of natural resources, where an individual (say, a fisherman or a lumberjack) decides upon extractive effort, anticipating that extracted resources will be sold on the market but will also reduce the stock available for the future. This situation can be described by model (50)-(52), where $S$ is the stock of natural resources, $\ell$ is extractive effort, $x=1-\ell$ is an alternative time use (say, leisure), and $y$ is a consumption good. Effort $\ell$ increases the flow of extracted resources $g(x, S)$ sold on the market at the exogenous price $w$, but it reduces the stock $S$. Since $\ell=1-x$, this means $g_{x}<0$ and $f_{x}>0$. These are exactly the key features of the training models discussed above, with only a difference in interpretation: in the present model of natural resources an increase in $x$ amounts to a reduction in extractive effort, whereas in those models of human capital it amounts to training. Hence, as above, if $r=\rho$, then Propositions 10 and 12 apply. In the present context it is plausible to assume that $f_{y}=0$, in which case Propositions 11 and Remark 6 apply. $^{26}$ If in steady state $f_{S} \in(0, \check{\rho})$, which is more likely the higher the degree of impatience, then an increase in the price of extracted resources leads to an initial increase and a long-run reduction in extractive effort, which is accompanied by a long-run reduction in the stock of natural resources.

\footnotetext{
${ }^{25}$ Formally, $\partial x^{s s} / \partial p>0$ if and only if $U_{y S}>-\left[\delta(\delta+\rho) U_{y y}+U_{S S}+\lambda_{0} w_{S S}\right] /(2 \delta+\rho)>0$.

${ }^{26}$ In this case the slope of the steady state Frisch supply of extractive effort is again $\frac{\partial \ell^{s s}}{\partial w}=$ $-\rho \lambda_{0} f_{S}\left[\left(f_{S}-\rho\right) g_{x}-f_{x} g_{S}\right] \check{\mathcal{H}}_{y y} /(|\check{J}| \check{\Omega})$.
} 


\section{Conclusion}

When consumers are forward-looking, price-based policies such as taxes and subsidies may affect demand in different ways in the short and in the long run, possibly generating 'perverse' effects over some time horizon. This paper studies short and long-run price effects for a broad class of dynamic consumer problems. We characterize the response of demand to a permanent price increase (both on impact and in steady state), as well as the longrun response of the relevant state variable. Price-based policies may indeed target either flow variables, such as consumption or schooling, or stock variables, such as human capital, health or natural resources, whose externalities are often the motivation for policy intervention.

The abstract setup we consider allows for the effect of past actions on current choices, for either static or dynamic budget constraints, and for resource allocation to consumption goods as well as for time allocation to labor and leisure. Our characterization provides results that are general, simple, widely applicable, empirically testable and policy relevant.

We show that both in the short and in the long run, price effects may be decomposed into the dynamic counterparts of income and substitution effect. Contrary to the case of Giffen goods in static consumer theory, we prove that, in response to a permanent price increase, demand can increase at some point in time even in the absence of income effects.

We find that short and long-run price effects may have opposite sign. This is due to the fact that consumers may face a trade-off between present and future consumption. Such trade-off essentially arises when the dynamic technology amplifies over time the effects of current choices on the relevant state variable, and this leads to counterbalancing choices in the future.

Under assumptions that are commonly used in the literature, this possibility materializes when a simple condition is satisfied, namely when the steady state impact of the state variable on its own speed of change is positive. This condition can be satisfied, for instance, in models of human capital accumulation, endogenous preferences or renewable resources, in which 'capital' is an input for its own accumulation.

After a permanent price increase, consumers with different time preferences react differently to the trade-off between present and future consumption created by such dynamic amplifier. Impatient consumers attach more weight to the present and reduce consumption today, accepting an increase tomorrow; patient consumers instead prefer to reduce consumption in the long run, and accept a short-run increase.

The above trade-off between present and future consumption does not arise when the dynamic technology acts as a stabilizer, dampening over time the effects of current choices on the state variable, and therefore allowing the short and long-run response of demand to 
a price increase to have the same sign. Under the common assumptions mentioned above, this possibility arises when the steady state impact of the state variable on its own speed of change is negative, as it easily happens in models with capital depreciation. Yet, even when the short and long-run price elasticity have the same sign, we find that the initial consumer response to a price increase may overshoot relative to the long-run response: if the dynamic stabilization effect is weak, demand is more elastic in the short than in the long run, whereas if it is strong, the reverse is true.

We illustrate the relevance of our results by applying them to the workhorse models used in different fields of economic research. We consider intertemporal models of human capital accumulation, addiction, habit and taste formation, health, and exploitation of natural resources. In each case, we show how price-based policies may have non-trivial effects over time. Our theoretical predictions, and in particular the non-monotonicity of the response of demand over time to a permanent price increase, can in principle be empirically tested in each of these domains.

Our framework can be extended to incorporate additional state and control variables, which may produce oscillatory and cyclical dynamic patterns (Becker and Murphy, 1988; Dockner and Feichtinger, 1993; Wirl, 1996). As intertemporal consumer problems are a building block of dynamic macroeconomic models, our results are also relevant for business cycle and growth theories, although an explicit analysis of general equilibrium models, possibly with a stochastic component, is outside the scope of the present paper.

\section{A Appendix}

\section{A.1 Illustrative example}

The steady state values of the illustrative example are found solving (8) and (9) and using the budget constraint (3) and FOC (5):

$$
\begin{aligned}
& x^{s s}=S^{s s}=\frac{(\rho-1)(1-p)-1}{|J|} \\
& \mu^{s s}=\frac{p}{|J|}, \quad y^{s s}=M-p x^{s s} .
\end{aligned}
$$

The Jacobian matrix associated to (8) and (9) is

$$
J=\left(\begin{array}{cc}
\rho-1 & -1 \\
-1 & 1
\end{array}\right) .
$$


Denote by $\varepsilon=\left(\rho-\sqrt{8-4 \rho+\rho^{2}}\right) / 2<0$ the smaller eigenvalue of $J$, and by $\xi=(1-\varepsilon, 1)$ the associated eigenvector. Note that neither $|J|=\rho-2$, nor $\varepsilon$ depend on the market price, and that stability requires $\rho<2$, which is assumed to hold hereafter.

Solving the system of linear ordinary differential equations (8) and (9), given $S(0)=S_{0}$, $S(\infty)=S^{s s}$ and $x(\infty)=x^{s s}$, yields

$$
\begin{aligned}
& x(t)=x^{s s}+\left(S_{0}-S^{s s}\right)(1-\varepsilon) \mathrm{e}^{\varepsilon t} \\
& S(t)=S^{s s}+\left(S_{0}-S^{s s}\right) \mathrm{e}^{\varepsilon t}
\end{aligned}
$$

Rearrange (60) and (61) to obtain the policy function:

$$
x(S)=x^{s s}+(1-\varepsilon)\left(S-S^{s s}\right) .
$$

After an increase in $p$ the whole policy function shifts downward when $\rho>1$, and upward when $\rho<1$, as

$$
\frac{\partial x(S)}{\partial p}=\varepsilon \frac{1-\rho}{|J|}
$$

The steady state response of $x$ to an increase in $p$ is:

$$
\frac{\partial x^{s s}}{\partial p}=\frac{1-\rho}{|J|}=\frac{1}{\varepsilon} \frac{\partial x(S)}{\partial p} .
$$

Since $\varepsilon$ is negative, the responses of $x$ to $p$ on impact and in steady state have opposite sign. Income effects, instead, are nil both on impact and in steady state:

$$
\frac{\partial x(S)}{\partial M}=\frac{\partial x^{s s}}{\partial M}=0
$$

To understand the effect of a change in the market price $p$ along the transition to the new steady state, remember that FOC (5) requires

$$
U_{x}=\Pi
$$

where $\Pi:=p+\mu$ denotes the full price of $x$ (see, e.g., Becker and Murphy, 1988). Since the problem is additively separable, an increase in the full price leads to a decrease in $x$ : $\frac{\partial x}{\partial \Pi}=\frac{1}{U_{x x}}<0$. Yet, the full price does not increase monotonically in the market price $p$. To see this, consider the value of the costate variable along an optimal path leading to the steady state:

$$
\mu(S)=\mu^{s s}+(\varepsilon-1)\left(S-S^{s s}\right)
$$


Hence

$$
\Pi(S)=p+\mu^{s s}+(\varepsilon-1)\left(S-S^{s s}\right)
$$

and, in steady state,

$$
\Pi\left(S^{s s}\right)=p+\mu^{s s}
$$

On impact (i.e. given $S$ ), the response of $\Pi$ to a rise in $p$ is

$$
\frac{\partial \Pi(S)}{\partial p}=1+\frac{\partial \mu^{s s}}{\partial p}-(\varepsilon-1) \frac{\partial S^{s s}}{\partial p}=\frac{\varepsilon(\rho-1)}{|J|}
$$

and, in steady state,

$$
\frac{\partial \Pi^{s s}}{\partial p}=1+\frac{\partial \mu^{s s}}{\partial p}=\frac{\rho-1}{|J|}=\varepsilon \frac{\partial \Pi(S)}{\partial p} .
$$

Since $\varepsilon$ is negative, the full price response to $p$ on impact and in steady state have opposite sign. Over time, irrespective of the degree of impatience, the full price of $x$ decreases in $S$ : $\Pi^{\prime}(S)=\varepsilon-1<0$. Thus, when the agent is impatient $(\rho>1)$, after a permanent increase in $p$, $\Pi$ first rises and then progressively falls. In the new steady state, despite the fact that the market price $p$ is higher, the full price $\Pi$ is lower than in the initial steady state, which explains why $x^{s s}$ is higher. The reverse pattern holds for $\rho<1$.

\section{A.2 Static counterpart of the illustrative example}

For the sake of comparability, we present here the static counterpart of the illustrative example considered in Section 2. Given $S, p$ and $M$, the consumer solves the following problem:

$$
\begin{aligned}
\max _{x, y} U(x, y, S) & =\max _{x, y}\left(x-\frac{x^{2}}{2}+y+S-\frac{S^{2}}{2}\right) \\
\text { s.t. } \quad M & =p x+y .
\end{aligned}
$$

Let $p \in(0,1)$ and $M>p(1-p)$, so as to grant an internal solution. Replacing the budget constraint, the problem can be re-written in terms of $V(x ; S, p, M)=U(x, M-p x, S)$, with first order condition $V_{x}=1-x-p=0$. The corresponding optimal consumption choices are $x^{*}=1-p$ and $y^{*}=M-p(1-p)$. The quasi-linear specification of the utility function ensures that all income effects are captured by $y$ and that $\partial x^{*} / \partial M=0$, so that changes in $p$ only determine substitution effects on the demand for $x$ and $\frac{\partial x^{*}}{\partial p}=-1$. 


\section{A.3 Static consumer problem}

We now briefly remind well known results from static consumer theory, to emphasize that, in a static environment, violations of the law of demand require a strong and negative interaction between consumption goods in the utility function and cannot occur absent income effects.

Consider a utility function $U(x, y)$, where $x \geq 0$ and $y \geq 0$ are two consumption goods. The utility function is assumed to be concave, continuously differentiable and non satiated. Given income $M>0$ and market price $p>0$, the problem is the following

$$
\begin{array}{ll}
\max _{x, y} & U(x, y) \\
\text { s.t. } & M=p x+y .
\end{array}
$$

The Lagrangian function corresponding to this problem is

$$
\mathcal{L}=U(x, y)+\lambda[M-p x-y]
$$

where $\lambda$ is the Lagrangian multiplier associated to the static budget constraint.

In an internal solution the following FOCs hold:

$$
\begin{aligned}
\mathcal{L}_{x} & =U_{x}-p \lambda=0 \\
\mathcal{L}_{y} & =U_{y}-\lambda=0 \\
\mathcal{L}_{\lambda} & =M-p x-y=0
\end{aligned}
$$

which implicitly determine the optimal values $\hat{x}, \hat{y}$ and $\hat{\lambda}$ as functions of $p$ and $M$. As it is well known, applying the implicit function theorem, the static price effect is

$$
\frac{\partial \hat{x}}{\partial p}=-\frac{\hat{\lambda}}{\hat{\Omega}}-\hat{x} \frac{\partial \hat{x}}{\partial M}
$$

where $\hat{\Omega}=-U_{x x}+2 p U_{x y}-p^{2} U_{y y}>0$ by concavity. The first term of (80) represents the static substitution effect, which is negative under non satiation (see $\mathcal{L}_{x}$ and $\mathcal{L}_{y}$ ), while the second term represents the static income effect, with

$$
\frac{\partial \hat{x}}{\partial M}=\frac{U_{x y}-p U_{y y}}{\hat{\Omega}} .
$$

Hence $U_{x y}$, the interaction between $x$ and $y$ in the utility function, crucially determines the sign of the static income effect. If such interaction is sufficiently negative $\left(U_{x y}<p U_{y y}\right)$, 
good $x$ is inferior; if it is below an even lower threshold $\left(U_{x y}<-\hat{\lambda} / \hat{x}+p U_{y y}\right)$, good $x$ is a Giffen good and the law of demand is violated. The reason is that, at the optimum, the FOCs imply $M R S_{x y}=U_{x} / U_{y}=p$. When $p$ increases, for ordinary goods the $M R S$ is raised by consuming less $x$ and more $y$, thus reducing $U_{x}$ and increasing $U_{y}$. For Giffen goods, instead, the increase in the $M R S$ is indeed obtained by consuming more $x$ in response to an increase in its price, because increasing the consumption of $x$ reduces $U_{x}$, but it also reduces $U_{y}$ more than proportionally. With a quasi-linear utility function, such as the one assumed in Appendix A.2, $U_{x y}=U_{y y}=0$ and income effects are nil because $\partial \hat{x} / \partial M=0$.

\section{A.4 Dynamic consumer problem without saving}

We are interested in assessing the elements of (27) and (28), which we report here for convenience:

$$
\begin{aligned}
\frac{\mathrm{d} x\left(S^{s s}\right)}{\mathrm{d} p} & =\frac{\partial x^{*}}{\partial p}+\frac{\partial x^{*}}{\partial \mu} \frac{\mathrm{d} \mu\left(S^{s s}\right)}{\mathrm{d} p} \\
\frac{\partial x^{s s}}{\partial p} & =\frac{\partial x^{*}}{\partial p}+\frac{\partial x^{*}}{\partial S} \frac{\partial S^{s s}}{\partial p}+\frac{\partial x^{*}}{\partial \mu} \frac{\partial \mu^{s s}}{\partial p} .
\end{aligned}
$$

Let

$$
\Omega(\mathcal{H})=-\mathcal{H}_{x x}+2 p \mathcal{H}_{x y}-p^{2} \mathcal{H}_{y y}
$$

which is positive by concavity (with strict concavity in $x$ ). The determinant of the Jacobian matrix associated to (24)-(25) is

$$
\begin{aligned}
|J(\mathcal{H})| & =f_{S}\left(\rho-f_{S}\right)+\frac{f_{x}-p f_{y}}{\Omega(\mathcal{H})}\left[\left(2 f_{S}-\rho\right)\left(p \mathcal{H}_{y S}-\mathcal{H}_{x S}\right)+\left(f_{x}-p f_{y}\right) \mathcal{H}_{S S}\right] \\
& +\frac{w_{S}}{\Omega(\mathcal{H})}\left\{2\left(f_{x}-p f_{y}\right)\left(f_{x} \mathcal{H}_{y S}-f_{y} \mathcal{H}_{x S}\right)+\left[f_{x}\left(p \mathcal{H}_{y y}-\mathcal{H}_{x y}\right)+f_{y}\left(\mathcal{H}_{x x}-p \mathcal{H}_{x y}\right)\right]\left(2 f_{S}-\rho\right)\right\} \\
& +\frac{w_{S}^{2}}{\Omega(\mathcal{H})}\left(f_{y}^{2} \mathcal{H}_{x x}-2 f_{x} f_{y} \mathcal{H}_{x y}+f_{x}^{2} \mathcal{H}_{y y}\right)
\end{aligned}
$$

For ease of notation, in the following we omit the argument $\mathcal{H}$ and simply write $J$ and $\Omega$. The negative eigenvalue of $J$ is

$$
\varepsilon=\frac{\rho-\sqrt{\rho^{2}-4|J|}}{2}
$$

From the FOCs (16)-(18) one obtains:

$$
\frac{\partial x^{*}}{\partial p}=-\frac{\lambda^{*}}{\Omega}-x^{*} \frac{\partial x^{*}}{\partial M}
$$


where

$$
\frac{\partial x^{*}}{\partial M}=\frac{\mathcal{H}_{x y}-p \mathcal{H}_{y y}}{\Omega} .
$$

In general expression (87) is different from the static price effect (80) and from the overall price effect of a dynamic consumer's maximization problem (which we will compute below) because it identifies the slope of the demand function keeping the state and its shadow price (i.e. the costate) fixed. ${ }^{27}$ Moreover:

$$
\begin{aligned}
\frac{\partial x^{*}}{\partial S} & =\frac{\mathcal{H}_{x S}-p \mathcal{H}_{y S}}{\Omega}+w_{S} \frac{\mathcal{H}_{x y}-p \mathcal{H}_{y y}}{\Omega} \\
\frac{\partial x^{*}}{\partial \mu} & =\frac{f_{x}-p f_{y}}{\Omega} .
\end{aligned}
$$

The terms $\partial S^{s s} / \partial p$ and $\partial \mu^{s s} / \partial p$ can be computed by applying the implicit function theorem to (24)-(25), which yields:

$$
\begin{aligned}
\frac{\partial S^{s s}}{\partial p}= & -\lambda^{s s} \frac{f_{S}+f_{y} w_{S}-\rho}{|J|} \frac{\partial x^{*}}{\partial \mu}-x^{s s} \frac{\partial S^{s s}}{\partial M} \\
\frac{\partial \mu^{s s}}{\partial p}= & \frac{\lambda^{s s}}{|J|}\left(\mathcal{H}_{S S} \frac{\partial x^{*}}{\partial \mu}-f_{S} \frac{\partial x^{*}}{\partial S}\right)-x^{s s} \frac{\partial \mu^{s s}}{\partial M} \\
& -w_{S} \lambda^{s s} \frac{f_{y}\left(\mathcal{H}_{x S}+p \mathcal{H}_{y S}\right)-2 f_{x} \mathcal{H}_{y S}+\left(f_{y} \mathcal{H}_{x y}-f_{x} \mathcal{H}_{y y}\right) w_{S}}{\Omega|J|}
\end{aligned}
$$

where

$$
\begin{aligned}
\frac{\partial S^{S S}}{\partial M}= & \frac{1}{\Omega|J|}\left\{\left(f_{x}-p f_{y}\right)\left(f_{y} \mathcal{H}_{x S}-f_{x} \mathcal{H}_{y S}\right)+\left(f_{S}-\rho\right)\left[f_{y}\left(p \mathcal{H}_{x y}-\mathcal{H}_{x x}\right)+f_{x}\left(\mathcal{H}_{x y}-p \mathcal{H}_{y y}\right)\right]\right. \\
\frac{\partial \mu^{s s}}{\partial M}= & \frac{1}{\Omega|J|}\left\{f_{x}\left[f_{x}^{2} \mathcal{H}_{y y}-2 f_{x} f_{y} \mathcal{H}_{x y}+f_{y}^{2} \mathcal{H}_{x x}\right)\right. \\
& +f_{y}\left[\mathcal{H}_{x x} \mathcal{H}_{S S}+p\left(\mathcal{H}_{S S} \mathcal{H}_{y y}-\mathcal{H}_{x S}^{2}-p\left(\mathcal{H}_{S S}\right)\right]\right. \\
& \left.+f_{S}\left[\mathcal{H}_{x y} \mathcal{H}_{x S}-\mathcal{H}_{x S} \mathcal{H}_{y x}\right)\right] \\
& +w_{S}\left[f_{x}\left(\mathcal{H}_{x S}+p\left(\mathcal{H}_{y y}-\mathcal{H}_{x y} \mathcal{H}_{y S}-\mathcal{H}_{x S} \mathcal{H}_{y y}\right)\right]\right. \\
&
\end{aligned}
$$

\footnotetext{
${ }^{27}$ As for the dynamic price effects reported in Proposition 1 and for the static price effect reported in (80), expression (87) can be interpreted as the sum of substitution and income effects. Note that (87) takes into account the role of state and costate variables (which makes it different from the static price effect), but it takes them as given (which makes it different from the dynamic price effects). If $\mu=0$, then $\partial x^{*} / \partial p=\partial \hat{x} / \partial p$. If the evolution of the state variable is linear and additively separable in the controls $\left(f_{x x}=f_{y y}=f_{x y}=0\right)$, then $\partial x^{*} / \partial M=\partial \hat{x} / \partial M$.
} 
Rearranging yields expressions (30) in Proposition 1 and (31) in Proposition 3, where

$$
\begin{aligned}
\frac{\partial x^{s s}}{\partial M} & =\frac{\partial x^{*}}{\partial M}+\frac{\partial x^{*}}{\partial S} \frac{\partial S^{s s}}{\partial M}+\frac{\partial x^{*}}{\partial \mu} \frac{\partial \mu^{s s}}{\partial M}=\frac{1}{\Omega|J|}\left\{\left(f_{S}-\rho\right)\left[f_{y}\left(\mathcal{H}_{x S}-p \mathcal{H}_{y S}\right)-f_{S}\left(\mathcal{H}_{x y}-p \mathcal{H}_{y y}\right)\right]\right. \\
& +\left(f_{x}-p f_{y}\right)\left(f_{S} \mathcal{H}_{y S}-f_{y} \mathcal{H}_{S S}\right)+w_{S}\left[f_{y}^{2} \mathcal{H}_{x S}-f_{y}\left(f_{S} \mathcal{H}_{x y}+f_{x} \mathcal{H}_{y S}\right)+f_{S} f_{x} \mathcal{H}_{y y}\right] .
\end{aligned}
$$

To appreciate the role of interactions in the utility function in generating income effects and being a possible source of violation of the law of demand, let us focus on the steady state and consider the first order condition (16). Define the full price as $\Pi:=U_{x}-\mathcal{H}_{x}$. As in the illustrative example, FOC (16) requires $U_{x}=\Pi$ along the optimal path, although now $\Pi=\lambda p-\mu f_{x}$. Differentiate (16) to get $U_{x x} \mathrm{~d} x+U_{x y} \mathrm{~d} y+U_{x S} \mathrm{~d} S=\mathrm{d} \Pi$. If $U_{x y}=U_{x S}=0$, as assumed in the illustrative model, then along the optimal path $x$ is inversely related to П. Thus, in that model violations of the law of demand in steady state can only be due to the fact that $\Pi^{s s}$ is decreasing in $p$. In general, however, the law of demand can be violated in steady state even if $\Pi^{s s}$ is not decreasing in $p$, due to the fact that $U_{x y}$ and $U_{x S}$ can be different from zero. The role of $U_{x y}$ closely resembles the mechanism behind Giffen goods emphasized in Appendix A.3: in a static consumer problem violations of the law of demand are due to a sufficiently negative $U_{x y}$ (i.e. $U_{x y}<-\hat{\lambda} / \hat{x}+p U_{y y}$ ) which implies that, in response to a rise in $p, U_{x}$ increases through a rise in $x$ and a reduction in $y$. The role of $U_{x S}$ is instead specific to a dynamic environment.

Finally, to compute (82) we need $\mathrm{d} \mu\left(S^{s s}\right) / \mathrm{d} p$. First we determine $\mu(S)$, which depends on $\left(S^{s s}, \mu^{s s}\right)$, by taking a first-order linear expansion of (24)-(25) around the steady state:

$$
\left(\begin{array}{c}
\dot{\mu} \\
\dot{S}
\end{array}\right)=J\left(\begin{array}{c}
\mu-\mu^{s s} \\
S-S^{s s}
\end{array}\right) .
$$

The solution of this system of ordinary linear differential equations yields

$$
\mu(S)=\mu^{s s}+\xi\left(S-S^{s s}\right)
$$

where $(\xi, 1)$ is the eigenvector associated to the negative eigenvalue $\varepsilon$ of $J$. A price change occurring when the system is already in steady state implies: ${ }^{28}$

$$
\frac{\mathrm{d} \mu\left(S^{s s}\right)}{\mathrm{d} p}=\frac{\partial \mu^{s s}}{\partial p}-\xi \frac{\partial S^{s s}}{\partial p}
$$

\footnotetext{
${ }^{28}$ In principle the derivative of (97) by $p$ also includes the term $\frac{\partial \xi}{\partial p}\left(S-S^{s s}\right)$, which vanishes for $S=S^{s s}$.
} 
Rearranging (82) yields expression (29) and completes the proof of Proposition 1, where

$$
\frac{\mathrm{d} x\left(S^{s s}\right)}{\mathrm{d} M}=\frac{\partial x^{*}}{\partial M}+\frac{\partial x^{*}}{\partial \mu} \frac{\mathrm{d} \mu\left(S^{s s}\right)}{\mathrm{d} M}
$$

and

$$
\frac{\mathrm{d} \mu\left(S^{s s}\right)}{\mathrm{d} M}=\frac{\partial \mu^{s s}}{\partial M}-\xi \frac{\partial S^{s s}}{\partial M} .
$$

Under the conditions of Remark $2, \partial x^{*} / \partial M=\mathrm{d} x\left(S^{s s}\right) / \mathrm{d} M=\partial x^{s s} / \partial M=\partial S^{s s} / \partial M=0$ and the price responses on impact and in steady state are, respectively

$$
\begin{aligned}
\frac{\mathrm{d} x\left(S^{s s}\right)}{\mathrm{d} p} & =\frac{\lambda^{s s}}{\Omega(\mathcal{H})|J(\mathcal{H})|} \varepsilon\left(f_{S}-\rho\right)>0 \Leftrightarrow f_{S}>\rho \\
\frac{\partial x^{s s}}{\partial p} & =\frac{\lambda^{s s}}{\Omega(\mathcal{H})|J(\mathcal{H})|} f_{S}\left(f_{S}-\rho\right)>0 \Leftrightarrow f_{S} \in(0, \rho) \\
\frac{\partial S^{s s}}{\partial p} & =-\frac{\lambda^{s s}}{\Omega(\mathcal{H})|J(\mathcal{H})|} f_{x}\left(f_{S}-\rho\right)=-\frac{f_{x}}{f_{S}} \frac{\partial x^{s s}}{\partial p} .
\end{aligned}
$$

This proves Remarks 2 and 3, and the non monotonicity result stated for $f_{S}>0$ in the first part of Proposition 2. For $f_{S}<0, x$ decreases both on impact and in steady state. Demand then is more elastic on impact if $\varepsilon<f_{S}$, which holds if

$$
\begin{aligned}
\rho-2 f_{S} & <\sqrt{\rho^{2}-4|J|}=\sqrt{\left(\rho-2 f_{S}\right)^{2}+4 \frac{f_{x}^{2} \mathcal{H}_{S S}+f_{x} \mathcal{H}_{x S}\left(\rho-2 f_{S}\right)}{\mathcal{H}_{x x}}} \\
& \Leftrightarrow f_{x}^{2} \mathcal{H}_{S S}+f_{x} \mathcal{H}_{x S}\left(\rho-2 f_{S}\right)<0 .
\end{aligned}
$$

This completes the proof of Proposition 2.

\section{A.5 Dynamic consumer problem with saving}

\section{A.5.1 Endogenous interest rate}

The FOCs (36)-(37) determine the optimal value of $x, y$ as functions of the state and costate variables, of the market price and income:

$$
\begin{aligned}
& x^{*}=x^{*}(S, A, \mu, \lambda ; p, M) \\
& y^{*}=y^{*}(S, A, \mu, \lambda ; p, M) .
\end{aligned}
$$


Replacing $\left(x^{*}, y^{*}\right)$ in (38)-(41) yields the optimal state and costate dynamics

$$
\begin{aligned}
\dot{S} & =f\left(x^{*}, y^{*}, S\right) \\
\dot{A} & =r(A)+M+w(S)-p x^{*}-y^{*} \\
\dot{\mu} & =\rho \mu-\tilde{\mathcal{H}}_{S}\left(x^{*}, y^{*}, S, A, \mu, \lambda ; p, M\right) \\
\dot{\lambda} & =\lambda\left(\rho-r_{A}\right) .
\end{aligned}
$$

Proceeding as in the previous Section, steady state consumption of $x$ is

$$
x^{s s}=x^{*}\left(S^{s s}, A^{s s}, \mu^{s s}, \lambda^{s s} ; p, M\right) .
$$

The change of steady state consumption of $x$ after an increase in its price $p$ is

$$
\frac{\partial x^{s s}}{\partial p}=\frac{\partial x^{*}}{\partial p}+\frac{\partial x^{*}}{\partial S} \frac{\partial S^{s s}}{\partial p}+\frac{\partial x^{*}}{\partial A} \frac{\partial A^{s s}}{\partial p}+\frac{\partial x^{*}}{\partial \mu} \frac{\partial \mu^{s s}}{\partial p}+\frac{\partial x^{*}}{\partial \lambda} \frac{\partial \lambda^{s s}}{\partial p} .
$$

In steady state, the determinant of the Jacobian associated to (106)-(109), is

$$
\begin{aligned}
|\tilde{J}|= & \frac{\lambda^{s s} r_{A A}}{\tilde{\Omega}}\left(\tilde{\mathcal{H}}_{x x}-2 p \tilde{\mathcal{H}}_{x y}+p^{2} \tilde{\mathcal{H}}_{y y}\right) f_{S}\left(f_{S}-\rho\right) \\
& +\frac{\lambda^{s s} r_{A A}}{\tilde{\Omega}}\left(f_{x}-p f_{y}\right)\left[\left(2 f_{S}-\rho\right)\left(p \tilde{\mathcal{H}}_{y S}-\tilde{\mathcal{H}}_{x S}\right)+\left(f_{x}-p f_{y}\right) \tilde{\mathcal{H}}_{S S}\right] \\
& +w_{S} \frac{\lambda^{s s} r_{A A}}{\tilde{\Omega}}\left\{2\left(f_{x}-p f_{y}\right)\left(f_{x} \tilde{\mathcal{H}}_{y S}-f_{y} \tilde{\mathcal{H}}_{x S}\right)+\left[f_{x}\left(p \tilde{\mathcal{H}}_{y y}-\tilde{\mathcal{H}}_{x y}\right)+f_{y}\left(\tilde{\mathcal{H}}_{x x}-p \tilde{\mathcal{H}}_{x y}\right)\right]\left(2 f_{S}-\rho\right)\right\} \\
& +w_{S}^{2} \frac{\lambda^{s s} r_{A A}}{\tilde{\Omega}}\left(f_{y}^{2} \tilde{\mathcal{H}}_{x x}-2 f_{x} f_{y} \tilde{\mathcal{H}}_{x y}+f_{x}^{2} \tilde{\mathcal{H}}_{y y}\right)
\end{aligned}
$$

where and $\lambda^{s s}$ is the steady state value of the costate variable associated to the dynamic budget constraint and

$$
\tilde{\Omega}=\tilde{\mathcal{H}}_{x x} \tilde{\mathcal{H}}_{y y}-\tilde{\mathcal{H}}_{x y}^{2}
$$

which is positive by strict concavity. Note that

$$
|\tilde{J}|=\frac{\lambda^{s s} r_{A A}}{\tilde{\Omega}} \Omega(\tilde{\mathcal{H}})|J(\tilde{\mathcal{H}})|
$$


where $\Omega(\tilde{\mathcal{H}})$ and $|J(\tilde{\mathcal{H}})|$ are defined in equations (84) and (85), but now applied to $\tilde{\mathcal{H}}$ instead of $\mathcal{H}$. Application of Cramer's rule to (36)-(37) yields

$$
\begin{aligned}
\frac{\partial x^{*}}{\partial p} & =\frac{\lambda}{\tilde{\Omega}} \tilde{\mathcal{H}}_{y y} \\
\frac{\partial x^{*}}{\partial S} & =\frac{\tilde{\mathcal{H}}_{x y} \tilde{\mathcal{H}}_{y S}-\tilde{\mathcal{H}}_{x S} \tilde{\mathcal{H}}_{y y}}{\tilde{\Omega}} \\
\frac{\partial x^{*}}{\partial A} & =\frac{\partial x^{*}}{\partial M}=0 \\
\frac{\partial x^{*}}{\partial \mu} & =\frac{f_{y} \tilde{\mathcal{H}}_{x y}-f_{x} \tilde{\mathcal{H}}_{y y}}{\tilde{\Omega}} \\
\frac{\partial x^{*}}{\partial \lambda} & =\frac{p \tilde{\mathcal{H}}_{y y}-\tilde{\mathcal{H}}_{x y}}{\tilde{\Omega}} .
\end{aligned}
$$

Analogously, from (106)-(109), one derives

$$
\begin{aligned}
\frac{\partial S^{s s}}{\partial p}= & \frac{\left(\lambda^{s s}\right)^{2} r_{A A}}{\tilde{\Omega}|\tilde{J}|}\left(p f_{y}-f_{x}\right)\left(f_{S}-\rho+f_{y} w_{S}\right)-x^{s s} \frac{\partial S^{s s}}{\partial M} \\
\frac{\partial A^{s s}}{\partial p}= & 0 \\
\frac{\partial \mu^{s s}}{\partial p}= & -\frac{\left(\lambda^{s s}\right)^{2} r_{A A}}{\tilde{\Omega}|\tilde{J}|}\left\{\tilde{\mathcal{H}}_{S S}\left(p f_{y}-f_{x}\right)+f_{S}\left(\tilde{\mathcal{H}}_{x S}-p \tilde{\mathcal{H}}_{y S}\right)\right. \\
& \left.+\left[f_{S}\left(\tilde{\mathcal{H}}_{x y}-p \tilde{\mathcal{H}}_{y y}\right)-2 f_{x} \tilde{\mathcal{H}}_{y S}+f_{y}\left(\tilde{\mathcal{H}}_{x S}+p \tilde{\mathcal{H}}_{y S}\right)+w_{S}\left(f_{y} \tilde{\mathcal{H}}_{x y}-f_{x} \tilde{\mathcal{H}}_{y y}\right)\right] w_{S}\right\} \\
& -x^{s s} \frac{\partial \mu^{s s}}{\partial M} \\
\frac{\partial \lambda^{s s}}{\partial p}= & \frac{\left(\lambda^{s s}\right)^{2} r_{A A}}{\tilde{\Omega}|\tilde{J}|}\left\{f_{S}^{2}\left(\tilde{\mathcal{H}}_{x y}-p \tilde{\mathcal{H}}_{y y}\right)-p f_{y}\left(f_{y} \tilde{\mathcal{H}}_{S S}+\rho \tilde{\mathcal{H}}_{y S}\right)\right. \\
& -f_{S}\left[f_{y}\left(\tilde{\mathcal{H}}_{x S}-2 p \tilde{\mathcal{H}}_{y S}\right)+\rho\left(\tilde{\mathcal{H}}_{x y}-p \tilde{\mathcal{H}}_{y y}\right)\right]-f_{x}\left[\tilde{\mathcal{H}}_{y S}\left(f_{S}-\rho\right)-f_{y} \tilde{\mathcal{H}}_{S S}\right] \\
& \left.-\left[f_{y}^{2} \tilde{\mathcal{H}}_{x S}+f_{x} \tilde{\mathcal{H}}_{y y}\left(f_{S}-\rho\right)-f_{y}\left(f_{S} \tilde{\mathcal{H}}_{x y}+f_{x} \tilde{\mathcal{H}}_{y S}-\rho \tilde{\mathcal{H}}_{x y}\right)\right] w_{S}\right\}-x^{s s} \frac{\partial \lambda^{s s}}{\partial M}(123)
\end{aligned}
$$


and

$$
\begin{aligned}
& \frac{\partial S^{S S}}{\partial M}=\frac{\lambda^{s s} r_{A A}}{\tilde{\Omega}|\tilde{J}|}\left\{\left(f_{x}-p f_{y}\right)\left(f_{y} \mathcal{H}_{x S}-f_{x} \mathcal{H}_{y S}\right)\right. \\
& +\left(f_{S}-\rho\right)\left[f_{x}\left(\tilde{\mathcal{H}}_{x y}-p \tilde{\mathcal{H}}_{y y}\right)+f_{y}\left(p \tilde{\mathcal{H}}_{x y}-\tilde{\mathcal{H}}_{x x}\right)\right] \\
& \left.-w_{S}\left(f_{x}^{2} \tilde{\mathcal{H}}_{y y}-2 f_{x} f_{y} \tilde{\mathcal{H}}_{x y}+f_{y}^{2} \tilde{\mathcal{H}}_{x x}\right)\right\} \\
& \frac{\partial A^{S S}}{\partial M}=0 \\
& \frac{\partial \mu^{S S}}{\partial M}=\frac{\lambda^{s s} r_{A A}}{\tilde{\Omega}|\tilde{J}|}\left\{f_{x}\left[\tilde{\mathcal{H}}_{x S} \tilde{\mathcal{H}}_{y S}-\tilde{\mathcal{H}}_{x y} \tilde{\mathcal{H}}_{S S}+p\left(\tilde{\mathcal{H}}_{S S} \tilde{\mathcal{H}}_{y y}-\tilde{\mathcal{H}}_{y S}^{2}\right)\right]\right. \\
& +f_{y}\left[\tilde{\mathcal{H}}_{x x} \tilde{\mathcal{H}}_{S S}-\tilde{\mathcal{H}}_{x S}^{2}+p\left(\tilde{\mathcal{H}}_{x S} \tilde{\mathcal{H}}_{y S}-\tilde{\mathcal{H}}_{x y} \tilde{\mathcal{H}}_{S S}\right)\right] \\
& +f_{S}\left[\tilde{\mathcal{H}}_{x S} \tilde{\mathcal{H}}_{x y}-\tilde{\mathcal{H}}_{x x} \tilde{\mathcal{H}}_{y S}+p\left(\tilde{\mathcal{H}}_{x y} \tilde{\mathcal{H}}_{y S}-\tilde{\mathcal{H}}_{x S} \tilde{\mathcal{H}}_{y y}\right)\right] \\
& \left.+w_{S}\left[f_{x}\left(\tilde{\mathcal{H}}_{y y} \tilde{\mathcal{H}}_{x S}-\tilde{\mathcal{H}}_{x y} \tilde{\mathcal{H}}_{y S}\right)+f_{y}\left(\tilde{\mathcal{H}}_{x x} \tilde{\mathcal{H}}_{y S}-\tilde{\mathcal{H}}_{x y} \tilde{\mathcal{H}}_{x S}\right)-f_{S} \tilde{\Omega}\right]\right\} \text {. } \\
& \frac{\partial \lambda^{S S}}{\partial M}=\frac{\lambda^{s s} r_{A A}}{\tilde{\Omega}|\tilde{J}|}\left\{f_{y}^{2}\left(\tilde{\mathcal{H}}_{S S} \tilde{\mathcal{H}}_{x x}-\tilde{\mathcal{H}}_{x S}^{2}\right)+f_{x}^{2}\left(\tilde{\mathcal{H}}_{S S} \tilde{\mathcal{H}}_{y y}-\tilde{\mathcal{H}}_{y S}^{2}\right)+f_{S}\left(f_{S}-\rho\right) \tilde{\Omega}\right. \\
& +f_{y}\left[2 f_{x}\left(\tilde{\mathcal{H}}_{x S} \tilde{\mathcal{H}}_{y S}-\tilde{\mathcal{H}}_{S S} \tilde{\mathcal{H}}_{x y}\right)+\left(\tilde{\mathcal{H}}_{x S} \tilde{\mathcal{H}}_{x y}-\tilde{\mathcal{H}}_{x x} \tilde{\mathcal{H}}_{y S}\right)\left(2 f_{S}-\rho\right)\right] \\
& \left.+\rho f_{x}\left(\tilde{\mathcal{H}}_{x S} \tilde{\mathcal{H}}_{y y}-\tilde{\mathcal{H}}_{x y} \tilde{\mathcal{H}}_{y S}\right)+2 f_{x} f_{S}\left(\tilde{\mathcal{H}}_{x y} \tilde{\mathcal{H}}_{y S}-\tilde{\mathcal{H}}_{x S} \tilde{\mathcal{H}}_{y y}\right)\right\}
\end{aligned}
$$

Expression (120) is identical to (46) and proves Proposition 6.

We now focus on the case in which the Jacobian admits two negative eigenvalues, which ensures saddle point stability to the steady state. When this is the case, $|\tilde{J}|$ is strictly positive. Under this stability requirement the following obtains

$$
\frac{\partial x^{s s}}{\partial p}=\frac{\left(\lambda^{s s}\right)^{2} r_{A A}}{\tilde{\Omega}|\tilde{J}|}\left(f_{S}+f_{y} w_{S}\right)\left(f_{S}+f_{y} w_{S}-\rho\right)-x^{s s} \frac{\partial x^{s s}}{\partial M}
$$

where

$$
\begin{aligned}
\frac{\partial x^{s s}}{\partial M} & =\frac{\lambda^{s s} r_{A A}}{\tilde{\Omega}|\tilde{J}|}\left\{\left(f_{S}-\rho\right)\left[f_{y}\left(\tilde{\mathcal{H}}_{x S}-p \tilde{\mathcal{H}}_{y S}\right)-f_{S}\left(\tilde{\mathcal{H}}_{x y}-p \tilde{\mathcal{H}}_{y y}\right)\right]+\left(f_{x}-p f_{y}\right)\left(f_{S} \tilde{\mathcal{H}}_{y S}-f_{y} \tilde{\mathcal{H}}_{S S}\right)\right. \\
& \left.+w_{S}\left[f_{y}^{2} \tilde{\mathcal{H}}_{x S}-f_{y}\left(f_{S} \tilde{\mathcal{H}}_{x y}+f_{x} \tilde{\mathcal{H}}_{y S}\right)+f_{S} f_{x} \tilde{\mathcal{H}}_{y y}\right]\right\} .
\end{aligned}
$$

Expression (128) is identical to (44) and proves the second part of Proposition 4. Remark 4 follows by comparison between expressions (120) and (124) with (128) and (129), when $f_{y}=\tilde{H}_{y S}=\tilde{H}_{x y}=w_{S}=0$.

Note that, if from (114) one replaces $\lambda^{s s} r_{A A} /(\tilde{\Omega}|\tilde{J}|)=1 /(\Omega(\tilde{\mathcal{H}})|J(\tilde{\mathcal{H}})|)$ in the last two 
equations, the price effect can be written as

$$
\frac{\partial x^{s s}}{\partial p}=\frac{\lambda^{s s}}{\Omega(\tilde{\mathcal{H}})|J(\tilde{\mathcal{H}})|}\left(f_{S}+f_{y} w_{S}\right)\left(f_{S}+f_{y} w_{S}-\rho\right)-x^{s s} \frac{\partial x^{s s}}{\partial M}
$$

which is identical to (30), except for the fact that the Hamiltonian function $\mathcal{H}$ of the problem without saving is replaced with the Hamiltonian function $\tilde{\mathcal{H}}$ of the problem with saving, and that $\lambda^{s s}$ now refers to the steady state value of the costate associated to the dynamic budget constraint. With the same caveat, an analogous replacement in (129) yields an expression identical to (95). ${ }^{29}$

To compute the response on impact

$$
\frac{\mathrm{d} x\left(S^{s s}, A^{s s}\right)}{\mathrm{d} p}=\frac{\partial x^{*}}{\partial p}+\frac{\partial x^{*}}{\partial \mu} \frac{\mathrm{d} \mu\left(S^{s s}, A^{s s}\right)}{\mathrm{d} p}+\frac{\partial x^{*}}{\partial \lambda} \frac{\mathrm{d} \lambda\left(S^{s s}, A^{s s}\right)}{\mathrm{d} p}
$$

we proceed as in the previous Section. Take a first-order linear expansion of (106)-(109) around the steady state:

$$
\left(\begin{array}{c}
\dot{S} \\
\dot{A} \\
\dot{\mu} \\
\dot{\lambda}
\end{array}\right)=\tilde{J}\left(\begin{array}{c}
S-S^{s s} \\
A-A^{s s} \\
\mu-\mu^{s s} \\
\lambda-\lambda^{s s}
\end{array}\right)
$$

Let $\left(\xi_{1}, \xi_{2}, \xi_{3}, \xi_{4}\right)$ and $\left(\omega_{1}, \omega_{2}, \omega_{3}, \omega_{4}\right)$ be the eigenvectors associated to the negative eigenvalues $\varepsilon_{1}$ and $\varepsilon_{2}$ of the Jacobian matrix $\tilde{J}$, which we do not report here for the sake of exposition. The solution of the above system of ordinary linear differential equations yields

$$
\begin{aligned}
& \mu(S, A)=\mu^{s s}+\zeta_{1}\left(S-S^{s s}\right)+\zeta_{2}\left(A-A^{s s}\right) \\
& \lambda(S, A)=\lambda^{s s}+\zeta_{3}\left(S-S^{s s}\right)+\zeta_{4}\left(A-A^{s s}\right)
\end{aligned}
$$

where $\zeta_{1}=\left(\omega_{3} \xi_{2}-\omega_{2} \xi_{3}\right) / \theta, \zeta_{2}=\left(\omega_{1} \xi_{3}-\omega_{3} \xi_{1}\right) / \theta, \zeta_{3}=\left(\omega_{4} \xi_{2}-\omega_{2} \xi_{4}\right) / \theta, \zeta_{1}=\left(\omega_{1} \xi_{4}-\omega_{4} \xi_{1}\right) / \theta$, and $\theta=\omega_{1} \xi_{2}-\omega_{2} \xi_{1}$. Since $\partial A^{s s} / \partial p=0$, a price change occurring when the system is already in steady state implies:

$$
\begin{aligned}
& \frac{\mathrm{d} \mu\left(S^{s s}, A^{s s}\right)}{\mathrm{d} p}=\frac{\partial \mu^{s s}}{\partial p}-\zeta_{1} \frac{\partial S^{s s}}{\partial p} \\
& \frac{\mathrm{d} \lambda\left(S^{s s}, A^{s s}\right)}{\mathrm{d} p}=\frac{\partial \lambda^{s s}}{\partial p}-\zeta_{3} \frac{\partial S^{s s}}{\partial p} .
\end{aligned}
$$

\footnotetext{
${ }^{29}$ The same is true for expressions (120), (122), (124), (126), which, with the same caveat, are identical to (91), (92), (93), (94), respectively.
} 
Replacing in (131) and rearranging yields $\mathrm{d} x\left(S^{s s}, A^{s s}\right) / \mathrm{d} p$ as in equation (44), where, exploiting $\partial x^{*} / \partial M=\partial A^{s s} / \partial M=0$ and remembering that on impact $A$ and $S$ are given,

$$
\begin{aligned}
\frac{\mathrm{d} x\left(S^{s s}, A^{s s}\right)}{\mathrm{d} M} & =\frac{\partial x^{*}}{\partial \mu} \frac{\mathrm{d} \mu\left(S^{s s}, A^{s s}\right)}{\mathrm{d} M}+\frac{\partial x^{*}}{\partial \lambda} \frac{\mathrm{d} \lambda\left(S^{s s}, A^{s s}\right)}{\mathrm{d} M} \\
\tilde{\varepsilon} & =f_{S}+f_{y} w_{S}+\left(f_{x}-p f_{y}\right)\left(\frac{\partial x^{*}}{\partial S}+\zeta_{1} \frac{\partial x^{*}}{\partial \mu}+\zeta_{3} \frac{\partial x^{*}}{\partial \lambda}\right)
\end{aligned}
$$

and

$$
\begin{aligned}
& \frac{\mathrm{d} \mu\left(S^{s s}, A^{s s}\right)}{\mathrm{d} M}=\frac{\partial \mu^{s s}}{\partial M}-\zeta_{1} \frac{\partial S^{s s}}{\partial M} \\
& \frac{\mathrm{d} \lambda\left(S^{s s}, A^{s s}\right)}{\mathrm{d} M}=\frac{\partial \lambda^{s s}}{\partial M}-\zeta_{3} \frac{\partial S^{s s}}{\partial M} .
\end{aligned}
$$

This completes the proof of Proposition 4.

In a stable steady state of problem (32)-(34), if $f_{y}=\tilde{\mathcal{H}}_{y S}=\tilde{\mathcal{H}}_{x y}=w_{S}=0$, then the price effect on impact and in steady state become

$$
\begin{aligned}
\frac{\mathrm{d} x\left(S^{s s}, A^{s s}\right)}{\mathrm{d} p} & =\frac{\lambda^{s s} r_{A A}}{\tilde{\Omega}|\tilde{J}|}\left(\lambda^{s s}-p \tilde{\mathcal{H}}_{y y} x^{s s}\right) \tilde{\varepsilon}\left(f_{S}-\rho\right) \\
\frac{\partial x^{s s}}{\partial p} & =\frac{\lambda^{s s} r_{A A}}{\tilde{\Omega}|\tilde{J}|}\left(\lambda^{s s}-p \tilde{\mathcal{H}}_{y y} x^{s s}\right) f_{S}\left(f_{S}-\rho\right)=\frac{f_{S}}{\tilde{\varepsilon}} \frac{\mathrm{d} x\left(S^{s s}, A^{s s}\right)}{\mathrm{d} p} \\
\frac{\partial S^{s s}}{\partial p} & =-\frac{\lambda^{s s} r_{A A}}{\tilde{\Omega}|\tilde{J}|}\left(\lambda^{s s}-p \tilde{\mathcal{H}}_{y y} x^{s s}\right) f_{x}\left(f_{S}-\rho\right)=-\frac{f_{x}}{f_{S}} \frac{\partial x^{s s}}{\partial p}
\end{aligned}
$$

where

$$
\begin{aligned}
& \frac{\mathrm{d} x\left(S^{s s}, A^{s s}\right)}{\mathrm{d} M}=\frac{\lambda^{s s} r_{A A}}{\tilde{\Omega}|\tilde{J}|} p \tilde{\mathcal{H}}_{y y} \tilde{\varepsilon}\left(f_{S}-\rho\right) \\
& \frac{\partial x^{s s}}{\partial M}=\frac{\lambda^{s s} r_{A A}}{\tilde{\Omega}|\tilde{J}|} p \tilde{\mathcal{H}}_{y y} f_{S}\left(f_{S}-\rho\right)=\frac{f_{S}}{\tilde{\varepsilon}} \frac{\mathrm{d} x\left(S^{s s}, A^{s s}\right)}{\mathrm{d} M} \\
& \tilde{\varepsilon}=\frac{\varepsilon_{1} \varepsilon_{2}\left[f_{x}^{2} \tilde{\mathcal{H}}_{S S}+f_{x}\left(\rho-2 f_{S}\right) \tilde{\mathcal{H}}_{x S}+\left(\varepsilon_{1}+f_{S}-\rho\right)\left(\varepsilon_{2}+f_{S}-\rho\right) \tilde{\mathcal{H}}_{x x}\right]}{\left(\varepsilon_{1}+\varepsilon_{2}-f_{S}\right)\left(\varepsilon_{1}+f_{S}-\rho\right)\left(\varepsilon_{2}+f_{S}-\rho\right) \tilde{\mathcal{H}}_{x x}-f_{x}\left(f_{S}-\rho\right)\left[f_{x} \tilde{\mathcal{H}}_{S S}+\left(\rho-2 f_{S}\right) \tilde{\mathcal{H}}_{x S}\right]}
\end{aligned}
$$

By equation (37), condition $f_{y}=0$ ensures that $\lambda$ is positive along the optimal path (and hence in steady state); $|\tilde{J}|$ is positive in a steady state with saddle point stability, and $\tilde{\Omega}$ is positive by strict concavity. Based on numerical simulations, we conjecture that in any stable steady state $\tilde{\varepsilon}$ is negative, although we have no analytical proof to offer. If this is the case, the short and the long run response to a price increase have opposite sign whenever $f_{S}>0$. If instead $f_{S}<0$, both responses are negative and the demand is more elastic in 
the short run if $f_{S}>\tilde{\varepsilon}$. This proves Proposition 5 and Remark 4 .

\section{A.5.2 Exogenous interest rate}

When $r_{A}=r=\rho$, equation (38) implies that the shadow price of wealth is constant over time, $\lambda(t)=\lambda_{0}$ for all $t$, and the optimality conditions (36)-(41) become

$$
\begin{aligned}
\tilde{\mathcal{H}}_{x} & =U_{x}(x, y, S)-\lambda_{0} p+\mu f_{x}(x, y, S)=0 \\
\tilde{\mathcal{H}}_{y} & =U_{y}(x, y, S)-\lambda_{0}+\mu f_{y}(x, y, S)=0 \\
\dot{S} & =f(x, y, S) \\
\dot{A} & =r A+M+w(S)-p x-y \\
\dot{\mu} & =\rho \mu-\tilde{\mathcal{H}}_{S}\left(x, y, S, A, \mu, \lambda_{0} ; p, M\right) .
\end{aligned}
$$

Proceeding as in the previous Section allows obtaining the steady state consumption of $x$ :

$$
x^{s s}=x^{*}\left(S^{s s}, A^{s s}, \mu^{s s}, \lambda_{0} ; p, M\right)
$$

where function $x^{*}$ yields the value of $x$ that satisfies the first order conditions (147) and (148). The change of steady state consumption of $x$ after an increase in price $p$ is computed as follows:

$$
\frac{\partial x^{s s}}{\partial p}=\frac{\partial x^{*}}{\partial p}+\frac{\partial x^{*}}{\partial S} \frac{\partial S^{s s}}{\partial p}+\frac{\partial x^{*}}{\partial A} \frac{\partial A^{s s}}{\partial p}+\frac{\partial x^{*}}{\partial \mu} \frac{\partial \mu^{s s}}{\partial p}+\frac{\partial x^{*}}{\partial \lambda} \frac{\partial \lambda_{0}}{\partial p} .
$$

In general, $\lambda_{0}$ depends on the parameters of the model, possibly including market prices. When focusing on Frisch demand functions, however, price changes are compensated so that the marginal utility of wealth $\lambda_{0}$ remains constant. In this case, $\partial \lambda_{0} / \partial p$ is zero. In steady state, the determinant of the 3 -dimensional Jacobian matrix $J_{0}$ associated to (149)-(151), once the first order conditions are satisfied, is

$$
\begin{aligned}
\left|J_{0}\right|= & \rho f_{S}\left(\rho-f_{S}\right)+\frac{\rho}{\tilde{\Omega}}\left\{\left(f_{y} \tilde{\mathcal{H}}_{x S}-f_{x} \tilde{\mathcal{H}}_{y S}\right)^{2}-\left(f_{y}^{2} \tilde{\mathcal{H}}_{x x}-2 f_{x} f_{y} \tilde{\mathcal{H}}_{x y}+f_{x}^{2} \tilde{\mathcal{H}}_{y y}\right) \tilde{\mathcal{H}}_{S S}\right. \\
& \left.-\left(2 f_{S}-\rho\right)\left[\left(f_{y} \tilde{\mathcal{H}}_{x y}-f_{x} \tilde{\mathcal{H}}_{y y}\right) \tilde{\mathcal{H}}_{x S}+\left(f_{x} \tilde{\mathcal{H}}_{x y}-f_{y} \tilde{\mathcal{H}}_{x x}\right) \tilde{\mathcal{H}}_{y S}\right]\right\}
\end{aligned}
$$

where $\tilde{\Omega}$ is given by (113). To focus on stable steady states, we now consider the case in which the Jacobian matrix $J_{0}$ admits one negative eigenvalue $\varepsilon_{0}$ (there is at most one), implying $\left|J_{0}\right|<0$. The elements of equation (153) are given by equations (115)-(118) and 
by

$$
\begin{aligned}
\frac{\partial S^{s s}}{\partial p}= & \frac{\rho \lambda_{0}}{\tilde{\Omega}\left|J_{0}\right|}\left[f_{y}^{2} \tilde{\mathcal{H}}_{x S}+\left(f_{S}-\rho\right)\left(f_{x} \tilde{\mathcal{H}}_{y y}-f_{y} \tilde{\mathcal{H}}_{x y}\right)-f_{x} f_{y} \tilde{\mathcal{H}}_{y S}\right] \\
\frac{\partial A^{s s}}{\partial p}= & \frac{\lambda_{0}}{\tilde{\Omega}\left|J_{0}\right|}\left\{f_{S}\left(f_{S}-\rho\right)\left(\tilde{\mathcal{H}}_{x y}-p \tilde{\mathcal{H}}_{y y}\right)-f_{y}\left[p\left(f_{y} \tilde{\mathcal{H}}_{S S}+\rho \tilde{\mathcal{H}}_{y S}\right)+f_{S}\left(\tilde{\mathcal{H}}_{x S}-2 p \tilde{\mathcal{H}}_{y S}\right)\right]\right. \\
& \left.-f_{x}\left[\left(f_{S}-\rho\right) \tilde{\mathcal{H}}_{y S}-f_{y} \tilde{\mathcal{H}}_{S S}\right]-w_{S}\left[f_{y}^{2} \tilde{\mathcal{H}}_{x S}+\left(f_{S}-\rho\right)\left(f_{x} \tilde{\mathcal{H}}_{y y}-f_{y} \tilde{\mathcal{H}}_{x y}\right)-f_{y} f_{x} \tilde{\mathcal{H}}_{y S}\right]\right\} \\
& -x^{s s} \frac{\partial A^{s s}}{\partial M} \\
\frac{\partial \mu^{s s}}{\partial p}= & \frac{\rho \lambda_{0}}{\tilde{\Omega}\left|J_{0}\right|}\left[f_{S}\left(\tilde{\mathcal{H}}_{x S} \tilde{\mathcal{H}}_{y y}-\tilde{\mathcal{H}}_{x y} \tilde{\mathcal{H}}_{y S}\right)-f_{x}\left(\tilde{\mathcal{H}}_{S S} \tilde{\mathcal{H}}_{y y}-\tilde{\mathcal{H}}_{y S}^{2}\right)+f_{y}\left(\tilde{\mathcal{H}}_{x y} \tilde{\mathcal{H}}_{S S}-\tilde{\mathcal{H}}_{x S} \tilde{\mathcal{H}}_{y S}(1)\right] 57\right) \\
\frac{\partial \lambda_{0}}{\partial p}= & 0
\end{aligned}
$$

and

$$
\begin{aligned}
\frac{\partial A^{s s}}{\partial M}= & \frac{1}{\tilde{\Omega}\left|J_{0}\right|}\left\{f_{y}^{2}\left(\tilde{\mathcal{H}}_{S S} \tilde{\mathcal{H}}_{x x}-\tilde{\mathcal{H}}_{x S}^{2}\right)+f_{S}\left(f_{S}-\rho\right) \tilde{\Omega}+f_{x}^{2}\left(\tilde{\mathcal{H}}_{S S} \tilde{\mathcal{H}}_{y y}-\tilde{\mathcal{H}}_{y S}^{2}\right)\right. \\
& -f_{x}\left[2 f_{y}\left(\tilde{\mathcal{H}}_{S S} \tilde{\mathcal{H}}_{x y}-\tilde{\mathcal{H}}_{x S} \tilde{\mathcal{H}}_{y S}\right)-\left(2 f_{S}-\rho\right)\left(\tilde{\mathcal{H}}_{x y} \tilde{\mathcal{H}}_{y S}-\tilde{\mathcal{H}}_{x S} \tilde{\mathcal{H}}_{y y}\right)\right] \\
& \left.+f_{y}\left(2 f_{S}-\rho\right)\left(\tilde{\mathcal{H}}_{x S} \tilde{\mathcal{H}}_{x y}-\tilde{\mathcal{H}}_{x x} \tilde{\mathcal{H}}_{y S}\right)\right\} .
\end{aligned}
$$

Expression (155) is identical to (49) and thus proves Proposition 9.

Since $\partial x^{*} / \partial A=\partial x^{*} / \partial M=\partial S^{s s} / \partial M=\partial \lambda_{0} / \partial M=\partial \mu^{s s} / \partial M=0$, income effect at the steady state is nil:

$$
\frac{\partial x^{s s}}{\partial M}=\frac{\partial x^{*}}{\partial M}+\frac{\partial x^{*}}{\partial S} \frac{\partial S^{s s}}{\partial M}+\frac{\partial x^{*}}{\partial A} \frac{\partial A^{s s}}{\partial M}+\frac{\partial x^{*}}{\partial \lambda} \frac{\partial \lambda_{0}}{\partial M}+\frac{\partial x^{*}}{\partial \mu} \frac{\partial \mu^{s s}}{\partial M}=0 .
$$

Combining the above results and rearranging yields equation (48) in Proposition 7. Remark 5 follows by comparison between (48) and (49), when $f_{y}=0$.

Since $\partial x^{*} / \partial A=0$ and $\lambda$ is fixed, the response on impact is

$$
\frac{\mathrm{d} x\left(S^{s s}, A^{s s}\right)}{\mathrm{d} p}=\frac{\partial x^{*}}{\partial p}+\frac{\partial x^{*}}{\partial \mu} \frac{\mathrm{d} \mu\left(S^{s s}, A^{s s}\right)}{\mathrm{d} p} .
$$

Take a first-order linear expansion around the steady state of (149)-(151), once the FOCs are satisfied: 


$$
\left(\begin{array}{c}
\dot{S} \\
\dot{A} \\
\dot{\mu}
\end{array}\right)=\tilde{J}\left(\begin{array}{c}
S-S^{s s} \\
A-A^{s s} \\
\mu-\mu^{s s}
\end{array}\right)
$$

The solution of the above system of ordinary linear differential equations can be expressed as a function of the state variable $S$ only,

$$
\mu(S)=\mu^{s s}+\stackrel{\circ}{\xi}_{3}\left(S-S^{s s}\right)
$$

where $\left(1, \stackrel{\circ}{\xi}_{2}, \stackrel{\circ}{\xi}_{3}\right)$ is the eigenvector associated to the negative eigenvalue $\varepsilon_{0}$ of the Jacobian matrix $J_{0}$,

$$
\begin{aligned}
& \varepsilon_{0}=\frac{1}{2}\left(\rho-\sqrt{\rho^{2}-4 \frac{\left|J_{0}\right|}{\rho}}\right) \\
& \stackrel{\dot{\xi}}{3}=\frac{1}{\Phi}\left[\left(\varepsilon_{0}-f_{S}\right) \tilde{\Omega}+f_{x}\left(\tilde{\mathcal{H}}_{x S} \tilde{\mathcal{H}}_{y y}-\tilde{\mathcal{H}}_{x y} \tilde{\mathcal{H}}_{y S}\right)+f_{y}\left(\tilde{\mathcal{H}}_{x x} \tilde{\mathcal{H}}_{y S}-\tilde{\mathcal{H}}_{x S} \tilde{\mathcal{H}}_{x y}\right)\right]
\end{aligned}
$$

and

$$
\Phi=-f_{y}^{2} \tilde{\mathcal{H}}_{x x}+2 f_{x} f_{y} \tilde{\mathcal{H}}_{x y}-f_{x}^{2} \tilde{\mathcal{H}}_{y y}>0
$$

A price change occurring when the state variable $S$ is already in steady state implies, for any $A$ :

$$
\frac{\mathrm{d} \mu\left(S^{s s}\right)}{\mathrm{d} p}=\frac{\partial \mu^{s s}}{\partial p}-\stackrel{\circ}{\xi}_{3} \frac{\partial S^{s s}}{\partial p} .
$$

Rearranging (161), and noting that it does not depend on either $A$ or $M$, yields $\mathrm{d} x\left(S^{s s}\right) / \mathrm{d} p$ in expression (47) in Proposition 7.

If $f_{y}=0$, the response on impact and in steady state simplifies to

$$
\begin{aligned}
\frac{\mathrm{d} x\left(S^{s s}\right)}{\mathrm{d} p} & =-\frac{\rho \lambda_{0}}{\tilde{\Omega}\left|J_{0}\right|} \varepsilon_{0}\left(f_{S}-\rho\right) \tilde{\mathcal{H}}_{y y} \\
\frac{\partial x^{s s}}{\partial p} & =-\frac{\rho \lambda_{0}}{\tilde{\Omega}\left|J_{0}\right|} f_{S}\left(f_{S}-\rho\right) \tilde{\mathcal{H}}_{y y}=\frac{f_{S}}{\varepsilon_{0}} \frac{\mathrm{d} x\left(S^{s s}\right)}{\mathrm{d} p} \\
\frac{\partial S^{s s}}{\partial p} & =\frac{\rho \lambda_{0}}{\tilde{\Omega}\left|J_{0}\right|} f_{x}\left(f_{S}-\rho\right) \tilde{\mathcal{H}}_{y y}=\frac{f_{x}}{f_{S}} \frac{\partial x^{s s}}{\partial p}
\end{aligned}
$$

and Proposition 8 and Remark 5 follow. 


\section{A.6 Dynamic labor supply}

When $r=\rho, \lambda_{0}>0$ is constant over time and the optimality conditions are:

$$
\begin{aligned}
\check{\mathcal{H}}_{x} & =U_{x}(x, y, S)+\lambda_{0} w g_{x}(x, S)+\mu f_{x}(x, y, S)=0 \\
\check{\mathcal{H}}_{y} & =U_{y}(x, y, S)-\lambda_{0}+\mu f_{y}(x, y, S)=0 \\
\dot{S} & =f(x, y, S) \\
\dot{A} & =r A+M+w g(x, S)-y \\
\dot{\mu} & =\rho \mu-\check{\mathcal{H}}_{S}\left(x, y, S, A, \mu, \lambda_{0} ; w, M\right)
\end{aligned}
$$

with the transversality conditions $\lim _{t \rightarrow \infty} e^{-\rho t} \mu(t) S(t)=0$ and $\lim _{t \rightarrow \infty} e^{-\rho t} \lambda_{0} A(t)=0$. Proceeding as in the previous section allows one obtains

$$
x^{s s}=x^{*}\left(S^{s s}, A^{s s}, \mu^{s s}, \lambda_{0} ; w, M\right)
$$

and

$$
\frac{\partial x^{s s}}{\partial w}=\frac{\partial x^{*}}{\partial w}+\frac{\partial x^{*}}{\partial S} \frac{\partial S^{s s}}{\partial w}+\frac{\partial x^{*}}{\partial A} \frac{\partial A^{s s}}{\partial w}+\frac{\partial x^{*}}{\partial \lambda} \frac{\partial \lambda_{0}}{\partial w}+\frac{\partial x^{*}}{\partial \mu} \frac{\partial \mu^{s s}}{\partial w} .
$$

Let

$$
\begin{aligned}
\check{\Phi} & =-f_{y}^{2} \check{\mathcal{H}}_{x x}+2 f_{x} f_{y} \check{\mathcal{H}}_{x y}-f_{x}^{2} \check{\mathcal{H}}_{y y} \\
\check{\Omega} & =\check{\mathcal{H}}_{x x} \check{\mathcal{H}}_{y y}-\check{\mathcal{H}}_{x y}^{2}
\end{aligned}
$$

which are positive by strict concavity, and let

$$
\begin{aligned}
& |\check{J}|=\rho f_{S}\left(\rho-f_{S}\right)+\frac{\rho}{\tilde{\Omega}}\left\{\left(f_{y} \check{\mathcal{H}}_{x S}-f_{x} \check{\mathcal{H}}_{y S}\right)^{2}+\check{\Phi}^{\circ} \check{\mathcal{H}}_{S S}\right. \\
& \left.+\left(2 f_{S}-\rho\right)\left[\left(f_{x} \check{\mathcal{H}}_{y y}-f_{y} \check{\mathcal{H}}_{x y}\right) \check{\mathcal{H}}_{x S}+\left(f_{y} \check{\mathcal{H}}_{x x}-f_{x} \check{\mathcal{H}}_{x y}\right) \check{\mathcal{H}}_{y S}\right]\right\} \\
& \check{\varepsilon}=\frac{\rho}{2}-\frac{1}{2} \sqrt{\rho^{2}-4 \frac{|\check{J}|}{\rho}}
\end{aligned}
$$

be the determinant of the Jacobian matrix $\breve{J}$ at the steady state (which is the same function of $\check{\mathcal{H}}$ as $\left|J_{0}\right|$ in (154) is of $\tilde{\mathcal{H}}$ ), and the negative eigenvalue of $\breve{J}$, respectively. The elements 
of (177) are

$$
\begin{aligned}
\frac{\partial x^{*}}{\partial w} & =-\frac{\lambda_{0}}{\check{\Omega}_{x}} \check{\mathcal{H}}_{y y} \\
\frac{\partial x^{*}}{\partial S} & =\frac{\check{\mathcal{H}}_{x y} \check{\mathcal{H}}_{y S}-\check{\mathcal{H}}_{x S} \check{\mathcal{H}}_{y y}}{\check{\Omega}} \\
\frac{\partial x^{*}}{\partial A} & =0 \\
\frac{\partial x^{*}}{\partial \mu} & =\frac{f_{y} \check{\mathcal{H}}_{x y}-f_{x} \check{\mathcal{H}}_{y y}}{\check{\Omega}} \\
\frac{\partial x^{*}}{\partial \lambda} & =-\frac{w g_{x} \check{\mathcal{H}}_{y y}+\check{\mathcal{H}}_{x y}}{\check{\Omega}}
\end{aligned}
$$

and

$$
\begin{aligned}
\frac{\partial S^{s s}}{\partial w}= & -\frac{\rho \lambda_{0}}{\check{\Omega}|\check{J}|}\left\{g_{S} \check{\Phi}+g_{x}\left[\left(f_{S}-\rho\right)\left(f_{x} \check{\mathcal{H}}_{y y}-f_{y} \check{\mathcal{H}}_{x y}\right)+f_{y}^{2} \check{\mathcal{H}}_{x S}-f_{x} f_{y} \check{\mathcal{H}}_{y S}\right]\right\} \\
\frac{\partial \mu^{s s}}{\partial w}= & \frac{\rho \lambda_{0}}{\check{\Omega}|\check{J}|}\left\{g_{x}\left[f_{S}\left(\check{\mathcal{H}}_{x y} \check{\mathcal{H}}_{y S}-\check{\mathcal{H}}_{x S} \check{\mathcal{H}}_{y y}\right)+f_{x}\left(\check{\mathcal{H}}_{S S} \check{\mathcal{H}}_{y y}-\check{\mathcal{H}}_{y S}^{2}\right)+f_{y}\left(\check{\mathcal{H}}_{x S} \check{\mathcal{H}}_{y S}-\check{\mathcal{H}}_{x y} \check{\mathcal{H}}_{S S}\right)\right]\right. \\
& \left.+g_{S}\left[f_{S} \check{\Omega}+f_{x}\left(\check{\mathcal{H}}_{x y} \check{\mathcal{H}}_{y S}-\check{\mathcal{H}}_{x S} \check{\mathcal{H}}_{y y}\right)+f_{y}\left(\check{\mathcal{H}}_{x S} \check{\mathcal{H}}_{x y}-\check{\mathcal{H}}_{x x} \check{\mathcal{H}}_{y S}\right)\right]\right\} .
\end{aligned}
$$

As for the Frisch demand function, $\frac{\partial \lambda_{0}}{\partial w}=\frac{\partial x^{*}}{\partial A} \frac{\partial A^{s s}}{\partial w}=0$. Remembering that $\ell=1-x$, equation (55) follows. This proves the second part of Proposition 10. Expression (187) is identical to (56) and proves Proposition 12.

The computation of the response on impact follows the steps of the solution for the problem with saving and exogenous interest rate, yielding

$$
\begin{aligned}
\frac{\mathrm{d} x\left(S^{s s}\right)}{\mathrm{d} w}= & \frac{\lambda_{0}}{\check{\Phi}} f_{y}^{2} g_{x}+\frac{\rho \lambda_{0}}{\check{\Omega}|\check{J}|} \check{\varepsilon}\left(f_{y} \check{\mathcal{H}}_{x y}-f_{x} \check{\mathcal{H}}_{y y}\right)\left[g_{S}\right. \\
& \left.+g_{x} \frac{f_{y}\left(f_{y} \check{\mathcal{H}}_{x S}-f_{x} \check{\mathcal{H}}_{y S}\right)-\left(f_{y} \check{\mathcal{H}}_{x y}-f_{x} \check{\mathcal{H}}_{y y}\right)\left(f_{S}-\rho\right)}{\check{\Phi}}\right]
\end{aligned}
$$

and equation (54) follows, completing the proof of Proposition 10.

If $f_{y}=0$, the response on impact and in steady state simplifies to

$$
\begin{aligned}
\frac{\mathrm{d} x\left(S^{s s}\right)}{\mathrm{d} w} & =\frac{\rho \lambda_{0}}{\check{\Omega}|\check{J}|} \check{\mathcal{H}}_{y y} \check{\varepsilon}\left[g_{x}\left(f_{S}-\rho\right)-g_{S} f_{x}\right] \\
\frac{\partial x^{s s}}{\partial w} & =\frac{\rho \lambda_{0}}{\check{\Omega}|\check{J}|} \check{\mathcal{H}}_{y y} f_{S}\left[g_{x}\left(f_{S}-\rho\right)-g_{S} f_{x}\right]=\frac{f_{S}}{\check{\varepsilon}} \frac{\mathrm{d} x\left(S^{s s}\right)}{\mathrm{d} w} \\
\frac{\partial S^{s s}}{\partial w} & =-\frac{\rho \lambda_{0}}{\check{\Omega}|\check{J}|} \check{\mathcal{H}}_{y y} f_{x}\left[g_{x}\left(f_{S}-\rho\right)-g_{S} f_{x}\right]=-\frac{f_{x}}{f_{S}} \frac{\partial x^{s s}}{\partial w}
\end{aligned}
$$


and Proposition 11 and Remark 6 follow.

\section{References}

Abel, A. B. (1990). Asset prices under habit formation and catching up with the Joneses. American Economic Review, 38-42.

Arrow, K. J. (1962). The economic implications of learning by doing. Review of Economic Studies 29(3), 155-173.

Becker, G. S. and K. M. Murphy (1988). A theory of rational addiction. Journal of Political Economy 96(4), 675-700.

Ben-Porath, Y. (1967). The production of human capital and the life cycle of earnings. Journal of Political Economy 75 (4), 352-365.

Blinder, A. S. and Y. Weiss (1976). Human capital and labor supply: A synthesis. Journal of Political Economy 84(3), 449-72.

Caputo, M. R. (1990). Comparative dynamics via envelope methods in variational calculus. Review of Economic Studies 57(4), 689-697.

Caputo, M. R. (1997). The qualitative structure of a class of infinite horizon optimal control problems. Optimal Control Applications and Methods 18(3), 195-215.

Carroll, C. D., J. Overland, and D. N. Weil (2000). Saving and growth with habit formation. American Economic Review $90(3), 341-355$.

Clark, C. W., F. H. Clarke, and G. R. Munro (1979). The optimal exploitation of renewable resource stocks: problems of irreversible investment. Econometrica 47(1), 25-47.

Dockner, E. J. and G. Feichtinger (1993). Cyclical consumption patterns and rational addiction. The American Economic Review, 256-263.

Epstein, L. G. (1978). The Le Châtelier principle in optimal control problems. Journal of Economic Theory 19(1), 103-122.

Grossman, M. (1972). On the concept of health capital and the demand for health. Journal of Political Economy $80(2), 223-55$.

Heckman, J. (1974). Life cycle consumption and labor supply: An explanation of the relationship between income and consumption over the life cycle. American Economic Review 64(1), 188-194. 
Heckman, J. J. (1976). A life-cycle model of earnings, learning, and consumption. Journal of Political Economy 84(2), S9-S44.

Jensen, R. T. and N. H. Miller (2008). Giffen behavior and subsistence consumption. American Economic Review 98(4), 1553-1577.

Killingsworth, M. R. (1982). learning by doing and investment in training: A synthesis of two rival models of the life cycle. Review of Economic Studies 49(2), 263-271.

Mangasarian, O. L. (1966). Sufficient conditions for the optimal control of nonlinear systems. SIAM Journal on Control 4(1), 139-152.

Oniki, H. (1973). Comparative dynamics (sensitivity analysis) in optimal control theory. Journal of Economic Theory 6(3), 265-283.

Otani, K. (1982). Explicit formulae of comparative dynamics. International Economic Review 23(2), 411-419.

Seierstad, A. and K. Sydsaeter (1977). Sufficient conditions in optimal control theory. International Economic Review 18(2), 367-391.

Stigler, G. J. and G. S. Becker (1977). De gustibus non est disputandum. American Economic Review 67(2), 76-90.

Weiss, Y. (1972). On the optimal lifetime pattern of labour supply. Economic Journal 82(328), 1293-1315.

Wirl, F. (1996). Pathways to Hopf bifurcations in dynamic continuous-time optimization problems. Journal of Optimization Theory and Applications 91(2), 299-320.

Yaari, M. E. (1965). Uncertain lifetime, life insurance, and the theory of the consumer. Review of Economic Studies 32(2), 137-150. 


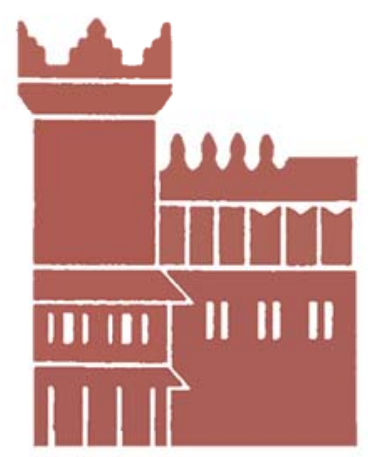

Alma Mater Studiorum - Università di Bologna DEPARTMENT OF ECONOMICS

Strada Maggiore 45

40125 Bologna - Italy

Tel. +39051 2092604

Fax +390512092664

http://www.dse.unibo.it 